\title{
Implications of a Fast Radio Burst from a Galactic Magnetar
}

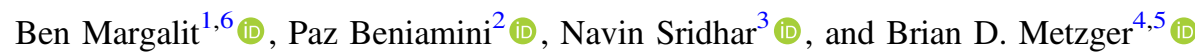 \\ ${ }^{1}$ Astronomy Department and Theoretical Astrophysics Center, University of California, Berkeley, Berkeley, CA 94720, USA; benmargalit@berkeley.edu \\ ${ }^{2}$ Division of Physics, Mathematics and Astronomy, California Institute of Technology, Pasadena, CA 91125, USA \\ ${ }^{3}$ Department of Astronomy, Columbia University, New York, NY 10027, USA \\ ${ }^{4}$ Columbia Astrophysics Laboratory, Columbia University, New York, NY 10027, USA \\ ${ }^{5}$ Center for Computational Astrophysics, Flatiron Institute, 162 W. 5th Ave., New York, NY 10011, USA \\ Received 2020 May 16; revised 2020 July 23; accepted 2020 August 5; published 2020 August 19
}

\begin{abstract}
A luminous radio burst was recently detected in temporal coincidence with a hard X-ray flare from the Galactic magnetar SGR 1935+2154 with a time and frequency structure consistent with cosmological fast radio bursts (FRBs) and a fluence within a factor of $\lesssim 10$ of the least energetic extragalactic FRB previously detected. Although active magnetars are commonly invoked FRB sources, several distinct mechanisms have been proposed for generating the radio emission that make different predictions for the accompanying higher-frequency radiation. We show that the properties of the coincident radio and X-ray flares from SGR 1935+2154, including their approximate simultaneity and relative fluence $E_{\text {radio }} / E_{\mathrm{X}} \sim 10^{-5}$, as well as the duration and spectrum of the X-ray emission, are consistent with extant predictions for the synchrotron maser shock model. Rather than arising from the inner magnetosphere, the X-rays are generated by (incoherent) synchrotron radiation from thermal electrons heated at the same internal shocks that produce the coherent maser emission as ultrarelativistic flare ejecta collides with a slower particle outflow (e.g., as generated by earlier flaring activity) on a radial scale of $\sim 10^{11} \mathrm{~cm}$. Although the rate of SGR 1935+2154-like bursts in the local universe is not sufficient to contribute appreciably to the extragalactic FRB rate, the inclusion of an additional population of more active magnetars with stronger magnetic fields than the Galactic population can explain both the FRB rate and the repeating fraction, but only if the population of active magnetars are born at a rate that is at least 2 orders of magnitude lower than that of the SGR 1935+2154-like magnetars. This may imply that the more active magnetar sources are not younger magnetars formed in a similar way to the Milky Way population (e.g., via ordinary supernovae) but are instead formed through more exotic channels, such as superluminous supernovae, accretion-induced collapse, or neutron star mergers.
\end{abstract}

Unified Astronomy Thesaurus concepts: Radio transient sources (2008); Magnetars (992); Soft gamma-ray repeaters (1471)

\section{Introduction}

Fast radio bursts (FRBs) are bright, millisecond-duration pulses of coherent radio emission with dispersion measures (DMs) well in excess of Galactic values (Lorimer et al. 2007; Thornton et al. 2013; see Cordes \& Chatterjee 2019; Petroff et al. 2019 for reviews) and hence pointing to an extragalactic origin. The precise mechanisms powering FRBs remain a topic of debate, in large part due to the small number of welllocalized events, as well as the fact that some FRBs appear to repeat (Spitler et al. 2016; Chatterjee et al. 2017; CHIME/FRB Collaboration et al. 2019), while others do not.

Many theoretical models have been proposed for FRBs (see Platts et al. 2019 for a catalog). Perhaps the most well-studied models are those that postulate that FRBs arise from the flaring activity of strongly magnetized neutron stars (NSs) known as "magnetars" (Popov \& Postnov 2013; Kulkarni et al. 2014; Lyubarsky 2014; Katz 2016; Beloborodov 2017; Kumar et al. 2017; Metzger et al. 2017). Evidence in favor of magnetars as FRB sources includes (1) high linear polarization and large rotation measures (e.g., Masui et al. 2015; Michilli et al. 2018), indicative of a strongly magnetized central engine and environment; (2) spatial association with star-forming regions in the two repeating events where very long baseline interferometry (VLBI) imaging enables precise sky localizations (Bassa et al. 2017;

\footnotetext{
${ }^{6}$ NASA Einstein Fellow.
}

Tendulkar et al. 2017; Marcote et al. 2020; although in a couple other cases, sources have been localized to galaxies with low global star formation); (3) statistical properties of the bursts' repetition consistent with those of Galactic magnetar flares (e.g., Wadiasingh \& Timokhin 2019; Cheng et al. 2020); and (4) a sufficiently high volumetric rate of magnetar birth to plausibly explain the observed FRB rate (e.g., Nicholl et al. 2017), unlike other models involving rare cataclysmic events (Ravi et al. 2019).

Despite these hints, several properties of the growing sample of FRBs appear, at least at first glance, to be in tension with magnetars as a primary source. The first repeating source, FRB 121102 (Spitler et al. 2016), has been bursting nearly continuously (albeit interrupted by extended "dark" periods) for over $7 \mathrm{yr}$; no known magnetar in our Galaxy matches this continuous level of activity. One is forced to the conclusion that at least the most active repeating FRB sources arise from magnetars that are somehow different from the Galactic population, e.g., being of very young age (Beloborodov 2017; Metzger et al. 2017), formed via alternative channels than ordinary core-collapse supernovae (CCSNe; Metzger et al. 2017; Margalit et al. 2019; Zhong \& Dai 2020), or possessing other atypical properties, such as an unusually long rotational period (Wadiasingh \& Timokhin 2019).

The recurrent FRB 180916 was recently shown by the CHIME/ FRB collaboration to exhibit a 16 day period of unknown origin 
(CHIME/FRB Collaboration et al. 2020a). Again, although known Galactic magnetars offer no clear explanation for periodic behavior at this scale (with the possible exception of candidate magnetar 1E 1613485055, which has a measured period of $6.7 \mathrm{hr}$; de Luca et al. 2006), reasonable variations in the properties of extragalactic magnetars (e.g., extremely slow rotation, precession, or presence in a binary) offer a potential explanation (Beniamini et al. 2020; Levin et al. 2020; Lyutikov et al. 2020; Tong et al. 2020; Yang \& Zou 2020; Zanazzi \& Lai 2020). Furthermore, a few FRBs have been localized to host galaxies with low levels of star formation (Bannister et al. 2019; Ravi 2019) uncharacteristic of the environments of magnetars in the Milky Way as being the product of relatively typical CCSNe, although more recent localizations show that the population as a whole remains broadly consistent with CCSN host galaxies (Bhandari et al. 2020).

Perhaps most challenging to the magnetar model, until recently, no Galactic magnetar had been observed to produce a radio burst with an energy matching that of known cosmological FRBs. An FRB-scale burst was ruled out from the giant magnetar flare in SGR 1806-20 insofar as it would have been detectable even in the side lobes of Parkes (Tendulkar et al. 2016). Such behavior may still be consistent with some magnetar models, e.g., because of beaming of the radio emission or because the nature of the external environment (i.e., the history of the flaring activity) plays as important a role in generating FRB emission as the flare itself (Beloborodov 2017, 2020; Metzger et al. 2019). ${ }^{7}$ Nevertheless, skepticism regarding magnetar FRB models would only continue to grow if FRBlike emission were never seen in association with nearby verifiable magnetars.

The observational situation changed abruptly with the recent discovery of a luminous millisecond radio burst from the Galactic magnetar SGR 1935+2154 (Bochenek et al. 2020c; CHIME/FRB Collaboration et al. 2020b). The double-peaked burst, detected independently by CHIME (Bandura et al. 2014) and STARE2 (Bochenek et al. 2020b), was temporally coincident with an X-ray burst of significantly larger fluence (Li et al. 2020; Mereghetti et al. 2020a; Ridnaia et al. 2020e; Tavani et al. 2020). This FRB is still a factor of $\sim 10$ less energetic than the weakest FRB previously detected from any localized cosmological FRB source. It nevertheless represents an enormous step in bridging the energy gap between Galactic magnetars and their hypothesized extragalactic brethren, providing new support to magnetar FRB models.

Here we explore several implications of this discovery for the broader magnetar-FRB connection. We emphasize that there is no single "magnetar model" but rather a range of models that make drastically different predictions for the mechanism and location of the radio emission and the accompanying higher-frequency radiation, some to which this discovery lends credence and others for which the model is placed in tension.

This paper is organized as follows. In Section 2 we summarize the observational picture regarding SGR 1935 +2154 and its recent radio/X-ray activity. In Section 3 we briefly discuss the SGR 1935+2154 FRB-producing burst in the context of other Galactic X-ray bursts. We continue by discussing the implications of this discovery for the population of extragalactic FRBs (Section 4). In Section 5 we discuss the

\footnotetext{
7 Another possibility that has been suggested in the context of magnetospheric models is that radio emission is quenched in more energetic magnetar flares due to inverse Compton losses (Katz 2020; although large electric fields, if attainable, may hinder this suppression, e.g., Baring et al. 2011).
}

implications of the coincident radio and X-ray flare from SGR $1935+2154$ for extant variations of the magnetar model. Finally, we summarize and provide bulleted conclusions in Section 6.

\section{Summary of Observations}

\section{1. $S G R 1935+2154$}

The Galactic soft gamma repeater (SGR) SGR 1935+2154 was first discovered by the Neil Gehrels Swift Observatory's Burst Alert Telescope (BAT) as a gamma-ray burst (GRB) candidate through a series of soft bursts from the Galactic plane (Lien et al. 2014; Stamatikos et al. 2014). The source is associated with supernova remnant (SNR) G57.2+0.8 (Gaensler 2014). Distance estimates are uncertain, ranging from 4.4 to $12.5 \mathrm{kpc}$ (Sun et al. 2011; Pavlović et al. 2013; Kothes et al. 2018; Mereghetti et al. 2020a; Zhou et al. 2020), and throughout this paper, we adopt a distance of $d=d_{10} 10 \mathrm{kpc}$. Subsequent discovery of the coherent X-ray pulsations of SGR $1935+2154$ with the Chandra X-ray Observatory established the spin period of the magnetar, $P \simeq$ $3.2 \mathrm{~s}$ (Israel et al. 2014, 2016). Observations of the source with XMM-Newton and NuSTAR during outburst in 2015 provided the magnetar's spin-down rate, $\dot{P} \simeq 1.43 \times 10^{-11} \mathrm{~s} \mathrm{~s}^{-1}$, which implies a surface dipolar magnetic field $B \simeq 2.2 \times 10^{14} \mathrm{G}$, spin-down luminosity $L_{\mathrm{sd}} \simeq 1.7 \times 10^{34} \mathrm{erg} \mathrm{s}^{-1}$, and characteristic spin-down age $P / 2 \dot{P} \simeq 3600 \mathrm{yr}$.

We note, however, that the age estimate based on the SNR association, $\gtrsim 16 \mathrm{kyr}$, is significantly older (Zhou et al. 2020; see also Kothes et al. 2018). This discrepancy between the dipolar and SNR age estimates is similar to the one observed in the other magnetar associated with an SNR, Swift J1834.9-0846 (Granot et al. 2017; with a spin-down age of $4.9 \mathrm{kyr}$ and an SNR age between 5 and $100 \mathrm{kyr}$ ). Since the dipolar age estimate is expected to be inaccurate in case either the surface magnetic field evolves with time (e.g., Colpi et al. 2000; Dall'Osso et al. 2012; Beniamini et al. 2019) or the spin evolution is not dominated by dipolar radiation (Thompson \& Blaes 1998; Harding et al. 1999; Beniamini et al. 2020), Granot et al. (2017) concluded that for Swift J1834.9-0846, the SNR age is likely to be the more realistic case. In this case, both Swift J1834.9-0846 and SGR 1935+2154 are significantly (by a factor of $\sim 4-10$ ) older than the majority of the observed Galactic magnetar population (Beniamini et al. 2019).

Radio observations with the Parkes telescope at 1.5 and $3 \mathrm{GHz}$ did not reveal any significant radio pulsations from SGR $1935+2154$ to a limiting flux of 0.1 and $0.07 \mathrm{mJy}$, respectively (Burgay et al. 2014). Observations with NCRA/GMRT and ORT at $\sim 300$ and $600 \mathrm{MHz}$ also found no radio pulses down to 0.4 and $0.2 \mathrm{mJy}$, respectively (Surnis et al. 2014, 2016), followed by additional 14 and $7 \mu \mathrm{Jy}$ limits by Arecibo (at 4.6 and $1.4 \mathrm{GHz}$; Younes et al. 2017).

The nondetection of periodic radio pulses from the source has not impaired SGR $1935+2154$ from being a prolific X-ray burster. This magnetar has been active since its discovery, with at least four outbursts on 2014 July 5, 2015 February 22, 2016 May 14, and 2016 June 18, each with an increasing number of bursts extending to higher energies (Lin et al. 2020a). During its active outburst cycles, SGR 1935+2154 exhibited a remarkably bright burst on 2015 April 12, detected by the four Interplanetary Network (IPN) spacecraft (Kozlova et al. 2016). This burst's long duration of $\sim 1.7 \mathrm{~s}$ and large fluence of $\sim 2.5 \times 10^{-5} \mathrm{erg} \mathrm{cm}^{-2}$ (radiated energy $\sim 10^{41} \mathrm{erg}$ ) place it in 
the rare class of "intermediate" SGR flares (Mazets et al. 1999; Feroci et al. 2003; Mereghetti et al. 2009; Göğüś et al. 2011).

The trend that the bursts from SGR 1935+2154 have become progressively more energetic in the years since its initial discovery (Younes et al. 2017) persists with the recent outburst, which is the most energetic yet (Mereghetti et al. 2020a; Zhang et al. 2020a). Similar behavior was previously observed for SGR 1806-20, which culminated with its production of the most energetic magnetar giant flare seen to date (Younes et al. 2015).

\subsection{An FRB from $S G R 1935+2154$}

On 2020 April 10, a short soft X-ray burst was triangulated by the IPN to SGR 1935+2154 (Svinkin et al. 2020). This was followed by a slew of bursts, extending to hard X-rays, detected over the following couple of weeks (Borghese et al. 2020; Cherry et al. 2020; Hurley et al. 2020; Kennea et al. 2020; Lipunov et al. 2020; Marathe et al. 2020; Mereghetti et al. 2020b; Palmer 2020; Ridnaia et al. 2020a, 2020b; Ricciarini et al. 2020; Ridnaia et al. 2020c, 2020d; Veres et al. 2020; Younes et al. 2020).

On April 28, as part of this period of enhanced source activity, a bright millisecond radio burst, the first of its kind, was detected from SGR 1935+2154 (Bochenek et al. 2020a, 2020c; Scholz \& CHIME/FRB Collaboration 2020; CHIME/ FRB Collaboration et al. 2020b). The radio burst was associated with a short hard X-ray burst (Mereghetti et al. 2020 b) peaking at energies $E_{\text {peak }} \sim 80 \mathrm{keV}$ (Li et al. 2020; Mereghetti et al. 2020a; Ridnaia et al. 2020e; Tavani et al. 2020). The detection by the CHIME/FRB back end in the $400-800 \mathrm{MHz}$ band comprises two subburst components. The bursts, each $\sim 0.5 \mathrm{~ms}$ wide and separated by $\sim 30 \mathrm{~ms}$, had a reported DM of $332.7 \mathrm{pc} \mathrm{cm}^{-3}$ (CHIME/FRB Collaboration et al. 2020b). This DM value is consistent with the observed $\approx 8.6 \mathrm{~s}$ delay of the radio burst (at $400 \mathrm{MHz}$ ) with respect to the peak of the X-ray counterpart flare as being almost entirely due to the cold plasma time delay (Mereghetti et al. 2020a, 2020b; Zhang et al. 2020b). An independent detection of the burst was also reported from the STARE2 radio feeds at the $1.4 \mathrm{GHz}$ band (Bochenek et al. 2020a, 2020c). They reported the burst arrival time and DM value to be consistent with the CHIME detection and constrained the peak fluence to be $\approx 1.5 \mathrm{MJy} \mathrm{ms}$.

The compelling nature of this burst led to a search for tracklike muon neutrino events with the IceCube observatory, with no significant neutrino signals detected along its direction (Vandenbroucke 2020). Likewise, Very Large Array follow-up of the source found no persistent or afterglow radio emission down to a flux of $\sim 50 \mu \mathrm{Jy}$ (Ravi et al. 2020a, 2020b).

The millisecond-duration high brightness temperature radio burst of SGR $1935+2154$ is unlike any other pulsar/magnetar phenomenology observed to date, with a luminosity exceeding that of even the most luminous Crab giant pulses (e.g., Mickaliger et al. 2012) by several orders of magnitude (Bochenek et al. 2020c; CHIME/FRB Collaboration et al. 2020b). Instead, the burst properties are suggestively similar to cosmological FRBs. As pointed out by Bochenek et al. (2020a), placed at the distance of the nearest localized FRB 180916, $\simeq 149$ Mpc (Marcote et al. 2020), the SGR 1935+2154 outburst would have been potentially detectable as a $\gtrsim 7 \mathrm{mJy} \mathrm{ms}$ burst, coming close to, albeit still lower than, typical FRB fluences. Stated energetically, SGR 1935's (isotropic-equivalent) emitted radio energy is $E_{\text {radio }} \approx 4 \times 10^{34} d_{10}^{2} \mathrm{erg}$, where we have normalized to a distance of $10 \mathrm{kpc} \times d_{10}$ and adopted the $1.5 \mathrm{MJy} \mathrm{ms}$ fluence and $B W \approx 250 \mathrm{MHz}$ bandwidth of STARE2 (Bochenek et al. 2020b, 2020c). ${ }^{8}$ The radio burst of SGR $1935+2154$ is thus within a factor of $\sim 10$ of the lowestenergy burst observed from any cosmological FRB of known distance to date, $\approx 5 \times 10^{35} \mathrm{erg}$ (Marcote et al. 2020). The immediate implication is that magnetar bursts akin to the SGR 1935+2154 April 28 event may be contributing to the extragalactic FRB population (Bochenek et al. 2020c; CHIME/FRB Collaboration et al. 2020b; see also Figure 3 and Section 4).

The fact that this Galactic FRB was associated with a contemporaneous X-ray counterpart exhibiting similar subburst temporal structure is an important diagnostic fact that we return to throughout this work. The radiated energy in this X-ray burst is $E_{\mathrm{X}} \approx 8 \times 10^{39} d_{10}^{2} \mathrm{erg}$, where we have adopted the $7 \times$ $10^{-7} \mathrm{erg} \mathrm{cm}^{-2}$ fluence reported by the Hard X-ray Modulation Telescope (HXMT; Li et al. 2020). A similar fluence was also reported by INTEGRAL (Mereghetti et al. 2020a), AGILE (Tavani et al. 2020), and Konus-Wind (Ridnaia et al. 2020e). The ratio of energy radiated in the radio and X-ray bands, or the radio "efficiency," is therefore

$$
\eta \equiv \frac{E_{\text {radio }}}{E_{\mathrm{X}}} \sim 10^{-5} \text {. }
$$

This single observation has dramatic implications. In the following section (Section 3), we use this property in discussing whether previous magnetar X-ray bursts may have produced similar FRBs that had gone undetected. In Section 4 we discuss the implications of the radio inefficiency implied by Equation (1) to the population of extragalactic FRBs. Finally, in Section 5 we show that the value of $\eta$ can serve as a diagnostic differentiating between theoretically proposed FRB models.

\section{The SGR 1935+2154 FRB in the Context of Galactic Magnetar Bursts}

In the following, we examine the burst from SGR 1935 +2154 more closely in light of other X-ray bursts from Galactic magnetars observed in the past $\sim 20 \mathrm{yr}$. Figure 1 depicts the X-ray fluence $\left(F_{X}\right)$ and duration $\left(t_{X}\right)$ of the recent X-ray bursts from SGR $1935+2154$ alongside other bright bursts. ${ }^{9}$ Here the duration of the burst $\left(t_{\mathrm{X}}\right)$ is defined as the interval of time when $5 \%$ and $95 \%$ of the total background subtracted counts are recorded (Göğüśs et al. 2001). A rough correlation is seen between the fluence and duration (Göğüś et al. 2001), approximately satisfying $F_{X} \propto t_{X}^{1.54}$, consistent with reports in the magnetar literature for the bursts from single sources (Gavriil et al. 2004). Although the bursts from SGR $1935+2154$ are bright, they fit within this trend and are not exceptional in terms of their fluence or overall envelope duration with respect to bright bursts from other Galactic magnetars. However, the burst associated with SGR 1935 +2154's radio emission may have exhibited a harder spectrum

\footnotetext{
8 The fact that the same burst was also detected by CHIME at lower radio frequencies suggests that its spectral energy distribution is broadband, such that the true burst energy could be larger by a factor of $\gtrsim \nu / B W \approx 6$.

9 The duration and fluence are obtained from an extensive search of the NASA GCN circulars archive (https://gcn.gsfc.nasa.gov/gcn3_archive.html) and the Astronomers' Telegram (http://astronomerstelegram.org/).
} 


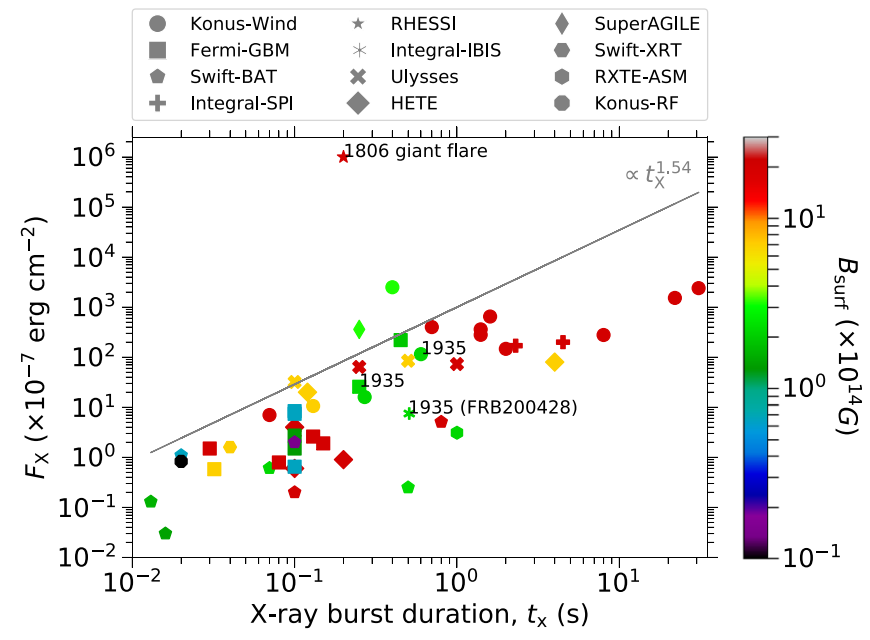

Figure 1. The X-ray fluence and duration of Galactic magnetar flares. The SGR $1935+2154$ flare associated with the April 28 radio burst (FRB 200428) is typical among Galactic magnetar flares. The gray line shows the fluenceduration correlation found by Gavriil et al. (2004), $F_{X} \propto t_{X}^{1.54}$. Points are colored based on the surface magnetic field of each magnetar (the McGill magnetar catalog, http://www.physics.mcgill.ca/pulsar/magnetar/main.html; Olausen \& Kaspi 2014), and the shape of each point denotes the detection instrument. A subsample of the X-ray flares detected during 2020 April from SGR $1935+2154$ is annotated against the respective points. In the event of a prolonged outburst (1-3 months), we show only the brightest burst of a given outburst. Likewise, for bursts clustered over timescales of a few seconds, we show the fluence corresponding to that of the initial peak, not the entire envelope of bursts. The anomalous concentration of bursts with $t_{\mathrm{X}} \sim 0.1 \mathrm{~s}$ can be attributed to instrument temporal resolution.

than other bursts (Mereghetti et al. 2020a), indicating that this particular burst may have been produced via a different emission mechanism, one intimately related to its FRB production. In Section 5.2.2 we present a scenario in which shock-powered $\mathrm{X}$-rays are generated concurrently with the coherent radio emission; however, as thermal X-rays may be produced in the magnetosphere during flaring by other mechanisms, it is plausible that multiple sources of X-ray emission contribute to different flares or within a single burst. Nonetheless, this raises the question of whether other FRBs from Galactic magnetars should have already been observed in the past.

Assuming the value of $\eta$ from the recent radio detection of SGR $1935+2154$ is universal across all flares, we predict the fluence of the radio emission ( $\left.F_{\text {radio }}\right)$ that should accompany the X-ray bursts from a large population of Galactic magnetar outbursts over the past $\sim 2$ decades (the same sample shown in Figure 1). Figure 2(a) shows $F_{\text {radio }}$ as a function of the burst date, with reference to the estimated sensitivity (hatched regions) of the STARE2 and CHIME/FRB telescopes. We see that two flares preceded the April 28 FRB event from SGR 1935+2154 with higher predicted $F_{\text {radio }}$ : one on 2020 April 10 (Veres et al. 2020) and another on 2020 April 22 (Ridnaia et al. 2020b). These events fall within the nominal STARE2 observable window, despite no reported radio detection. We note that relatively few magnetar flares have occurred over the last few years, when STARE2 and CHIME have been operational, at a fluence that would have been detectable by these instruments. Also note that the on-beam CHIME/FRB sensitivity shown is not the relevant one for most bursts, which will occur in the side lobes of the telescope.

In the exceptional case of the giant flare from SGR 1806-20 (Hurley et al. 2005; Palmer et al. 2005), the upper limit on the radio emission (Tendulkar et al. 2016; estimated based on the side-lobe sensitivity of Parkes) is smaller than the predicted radio fluence (for

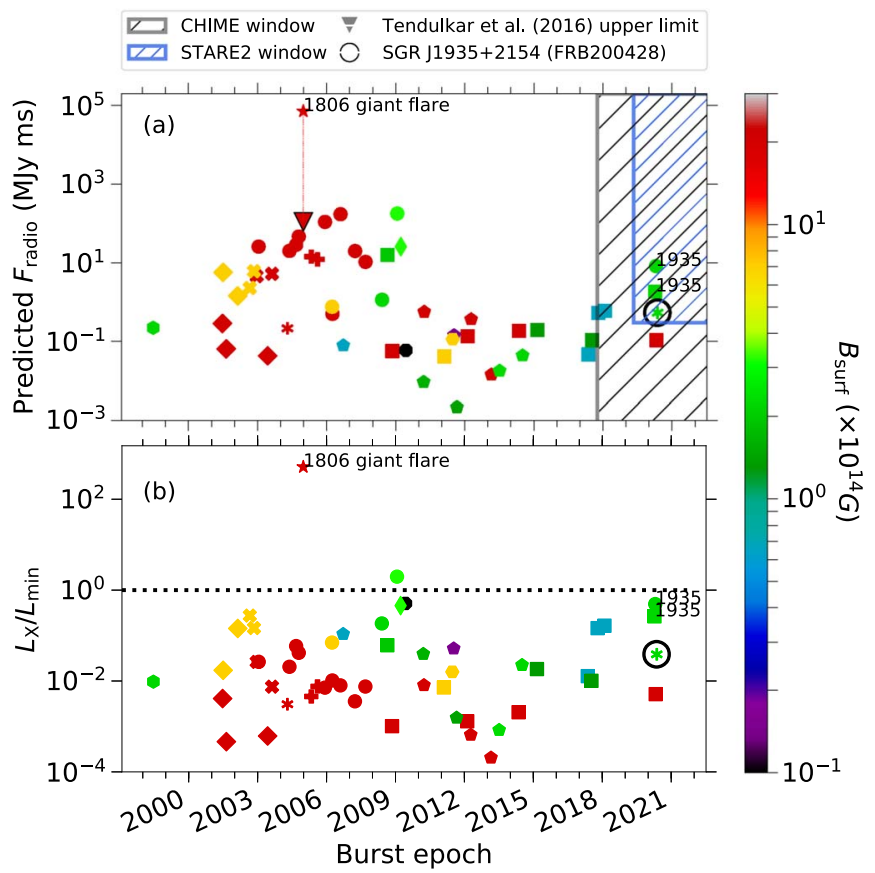

Figure 2. Properties of Galactic magnetar flares as a function of burst epoch. Different points denote the same set of sources and detection instruments as described in Figure 1. The point corresponding to the radio burst on April 28 (FRB 200428) is circled. (a) Predicted fluence of radio burst counterparts to magnetar flares, assuming a radio-to-X-ray efficiency $\eta \sim 10^{-5}$ (Equation (1)). Hatched blue and gray regions denote the observable windows of STARE2 and CHIME/FRB, respectively (assuming on-beam sensitivity limits). The upper limit on radio emission from the SGR 1806-20 giant flare (top red star) is shown as an upside-down triangle (Tendulkar et al. 2016). (b) Luminosity of the same bursts relative to the magnetic Eddington luminosity $L_{\min }$ (Paczynski 1992; see also Section 4), a rough scale for the minimum luminosity burst that is capable of driving baryonic outflows via radiation pressure.

$\eta \sim 10^{-5}$ ) by a factor of $\sim 100$ (Figure 2; red triangle and square, respectively). Monitoring of SGR $1935+2154$ by more sensitive radio facilities subsequent to the April 28 event has also shown that not every X-ray burst is accompanied by observable radio emission (Lin et al. 2020b) and supports suggestions that the radio emission may be related to the unusual spectrotemporal properties of the April 28 X-ray burst (Mereghetti et al. 2020a; Ridnaia et al. 2020e). As we discuss in Section 5.2.2, this may be understood in some magnetar FRB models for different beaming properties of the radio emission relative to the higher-frequency counterpart in giant flares, as opposed to less energetic magnetar flares. More generally, the fact that one FRB was observed out of only a few magnetar bursts in which we might have expected a detection suggests that the FRB beaming cannot be too extreme. The beaming can be directly probed in the future, once more X-ray bursts from SGR 1935 +2154 with comparable luminosities are observed, by searching for a possible correlation between FRB detectability and the rotational phase of the magnetar.

Using the X-ray burst fluence for each source and distance estimates from the McGill magnetar catalog (Olausen \& Kaspi 2014), ${ }^{10}$ we estimate the intrinsic burst X-ray luminosity $\left(L_{X}\right)$. For the selected bursts, we depict in Figure 2 the value of $L_{X}$ normalized to the individual source's magnetic Eddington luminosity $L_{\min }=$ $3.5 \times 10^{38}\left(B_{\text {surf }} / 10^{12} \mathrm{G}\right)^{4 / 3} \mathrm{erg} \mathrm{s}^{-1}$ (Paczynski 1992), where $B_{\text {surf }}$ is the surface dipole magnetic field strength, and $L_{\min }$ is a

\footnotetext{
${ }^{10}$ http://www.physics.mcgill.ca/ pulsar/magnetar/main.html
} 
rough scale for the minimum luminosity of a burst that can drive a mass outflow via radiation pressure.

Note that the FRB-producing flare from SGR 1935+2154 obeyed $L_{X}<L_{\min }$ (circled green symbol in Figure 2) and hence would not necessarily have been expected to produce a baryonloaded outflow, at least via radiation pressure. This is potentially relevant because some FRB models-particularly the baryonic shock models (Section 5.2.2) —require an upstream medium into which the shocks collide. On the other hand, other mechanisms than radiation pressure (e.g., magnetic stresses) may also play a role in mass ejection, and the quantity of mass in the external medium needed in the synchrotron maser scenario is extremely modest.

\section{Implications for the Extragalactic FRB Population}

In this section, we assume that all FRBs are produced by magnetar flares with universal properties motivated by the SGR 1935 burst. Proceeding under this strong assumption, we explore the implications for FRB energetics and repetition rates. We are led to conclude that "ordinary" magnetars with activity levels similar to SGR 1935 cannot alone explain the observed FRB population.

The low value of $\eta \sim 10^{-5}$ (Equation (1)) illustrates that magnetars are inefficient FRB producers. The implications of this fact for specific magnetar FRB models are discussed later (Section 5). Regardless of the emission mechanism, the active lifetime of cosmological recurrent FRB sources cannot be long if FRB emission is similarly inefficient for such sources. For the activity rate and radio fluences of FRB 121102 (e.g., Law et al. 2017), the radio inefficiency $\eta \sim 10^{-5}$ implies that the FRB-generating engine must be losing energy at a rate of $\sim$ several $\times 10^{39} \mathrm{erg} \mathrm{s}^{-1}$ (itself only a lower limit if the luminosity function is energetically dominated by low-energy undetectable bursts; Gourdji et al. 2019). For FRB 180916, the repetition rate and luminosity function point to qualitatively similar requirements on the power output of the central engine, $\gtrsim 5 \times 10^{38} \mathrm{erg} \mathrm{s}^{-1}$ (CHIME/FRB Collaboration et al. 2020a).

If recurrent FRBs are powered by magnetars, then their active lifetime is at the very least limited by their total magnetic energy reservoir, $E_{\mathrm{mag}} \sim 3 \times 10^{49} \mathrm{erg}\left(B / 10^{16}\right)^{2}$,

$$
\tau_{\text {active }} \sim \frac{E_{\mathrm{mag}}}{\dot{E}_{\mathrm{FRB}} / \eta} \sim 200 \mathrm{yr}\left(\frac{B}{10^{16} \mathrm{G}}\right)^{2}\left(\frac{\eta}{10^{-5}}\right)
$$

where $B$ is the interior magnetic field strength, and we have taken $\dot{E}_{\mathrm{FRB}} \sim 5 \times 10^{34} \mathrm{erg} \mathrm{s}^{-1}$ motivated by FRB 121102 (Law et al. 2017). Even for large interior fields, $B \gtrsim 10^{16} \mathrm{G}$, the maximum active lifetime is significantly shorter than the $16 \mathrm{kyr}$ estimated age of SGR $1935+2154$ (Zhou et al. 2020) and most other magnetars (although SGR 1806-20's age may in fact be comparable to that estimated in Equation (2); Tendulkar et al. 2012).

Based purely on their X-ray behavior, magnetars as active as FRB 121102 or other repeating FRB sources do not exist in our own Galaxy. These points suggest that if cosmological FRBs originate from magnetar progenitors, at least a subset of these magnetars must be far more active than SGR $1935+2154$ and are perhaps formed via different mechanisms than Galactic magnetars (Margalit et al. 2019). We further quantify this point

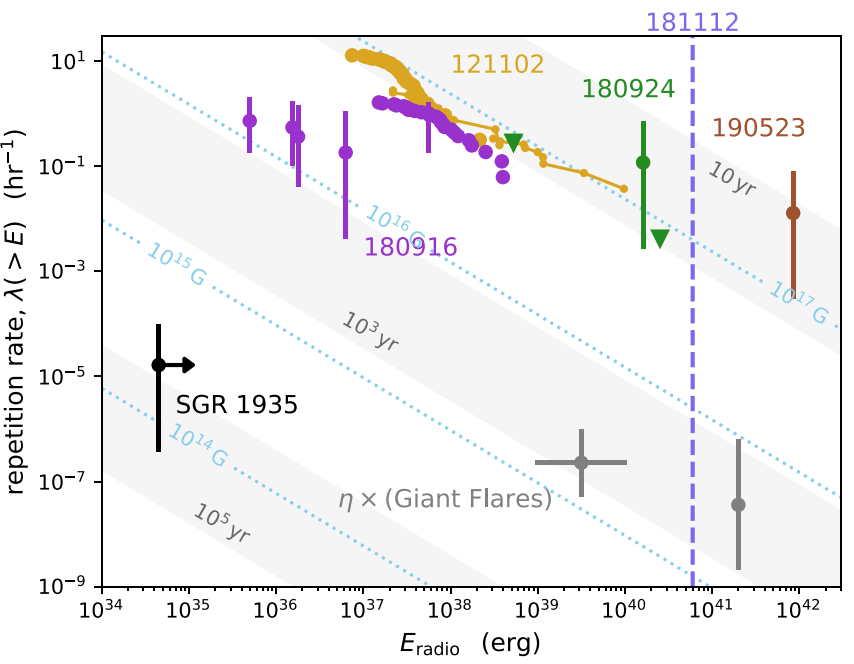

Figure 3. Repetition rate above a given emitted radio energy as a function of energy for SGR 1935+2154 and localized FRBs: the repeating sources FRB 121102 (Law et al. 2017; Gourdji et al. 2019) and 180916 (Marcote et al. 2020; CHIME/FRB Collaboration et al. 2020a) and apparently nonrepeating FRBs 180924 (Bannister et al. 2019), 190523 (Ravi 2019), and 181112 (Prochaska et al. 2019). The energy of the recent radio burst from SGR $1935+2154$ is a factor of $\sim 10 d_{10}^{2}$ lower than the least energetic extragalactic FRB of known distance. Applying the same ratio of radio to X-ray fluence measured for SGR $1935+2154 \eta \sim 10^{-5}$ (Equation (1)) to giant magnetar flares would imply that Galactic magnetars are capable of powering even the most energetic cosmological FRBs. However, a stark discrepancy exists between the activity (burst repetition rate) of Galactic magnetars and the sources of the recurring extragalactic FRBs (Section 4). Scaling from the magnetic field and age of SGR 1935+2154 implies that magnetar progenitors of extragalactic FRBs must have larger $B$ fields and younger ages (see Section 4.1 for further details)

in the next section by calculating the extragalactic detection rate of SGR $1935+2154-$ like events.

\subsection{Rates: A Single Magnetar Population?}

Given the 3.6 sr field of view of STARE2 (Bochenek et al. $2020 \mathrm{~b}$ ) and the fact that a single magnetar radio burst has been detected during the $\sim 300$ day operating period of the experiment, we estimate the rate of SGR 1935+2154-like magnetar radio bursts (taking the number of active magnetars in the Galaxy to be $N=29$; Olausen \& Kaspi 2014) to be $\lambda_{\text {mag }} \in[0.36,80] \times 10^{-2}$ magnetar $^{-1} \mathrm{yr}^{-1}$ at $95 \%$ confidence (assuming Poisson statistics; Gehrels 1986). ${ }^{11}$

The radio burst activity (repetition) rate of SGR 1935 estimated above is plotted in Figure 3 in comparison to cosmological FRBs. We show here the full sample of published localized FRB sources, where the radio-emitted (isotropicequivalent) energy can be reliably calculated: the repeating FRB 121102 (yellow; Law et al. 2017; Gourdji et al. 2019), the recently localized CHIME repeater FRB 180916 (purple; Marcote et al. 2020; CHIME/FRB Collaboration et al. 2020a), and the apparently nonrepeating FRBs 180924 (green, upper limits denoted by upside-down triangles; Bannister et al. 2019), 190523 (brown; Ravi 2019), and 181112 (blue; Prochaska et al. 2019). We have used quoted rates where possible (Law et al. 2017; Gourdji et al. 2019; CHIME/FRB Collaboration et al. 2020a) and otherwise estimated Poissonian rates based on quoted field exposure times where available.

\footnotetext{
${ }^{11}$ Bochenek et al. (2020c) derived a more accurate estimate of $\lambda_{\mathrm{mag}} \in[1.55$, $7.02] / N=[5,24] \times 10^{-2}$ magnetar $^{-1} \mathrm{yr}^{-1}$ at $68 \%$ confidence, whereas the CHIME/FRB Collaboration et al. (2020b) derived $\lambda_{\mathrm{mag}} \in[0.7,40] \times 10^{-2}$ magnetar ${ }^{-1} \mathrm{yr}^{-1}$, both consistent with our adopted range.
} 
Gray points with error bars show the repetition rate (from Tendulkar et al. 2016) and radio energy implied if some giant magnetar flares produce radio emission with the same efficiency $\eta \sim 10^{-5}$ between X-ray and radio fluences. This indicates that Galactic magnetars may be energetically capable of powering even the most luminous FRBs, though for one particular flare from SGR 1806-20, such radio emission is ruled out (Tendulkar et al. 2016). Finally, we note that radio pulses observed from M87 by Linscott \& Erkes (1980) 40 yr ago, though unconfirmed by subsequent follow-up, may have been the earliest recorded FRB detections and correspond to $E_{\text {radio }} \sim 10^{38}$ erg in this figure.

Figure 3 shows that, at comparable energy, the SGR 1935 radio burst is $\sim 10^{-5}$ less frequent than the FRB 180916 bursts. Within the hypothesis that magnetars are also the progenitors of such cosmological FRBs, this stark discrepancy in activity rate may be attributable to the magnetar age and internal field strength. Indeed, Margalit et al. (2019) showed that magnetar activity scales strongly with magnetic field, $\dot{E} \propto B^{3.2}$ (Dall'Osso et al. 2012; Beloborodov $\& \mathrm{Li} 2016) .{ }^{12}$ This is shown schematically with the dotted blue contours in Figure 3, assuming that $\lambda(>E) E \sim \dot{E} \propto B^{3.2}$, and scaling the $B$ field from $\sim 2 \times 10^{14} \mathrm{G}$, the external dipole field of SGR $1935+2154$ (note that the internal field is what sets $\dot{E}$, and thus we implicitly assume that the internal field is comparable to the external dipole field for this object). This assumed $B$ field implies an active lifetime of $\sim 70 \mathrm{kyr}$ for SGR 1935 (e.g., Margalit et al. 2019, their Equation (1)), broadly consistent with the $\gtrsim 16 \mathrm{kyr}$ source age. Contours of active lifetime ( magnetar age) are also shown in Figure 3 (gray shaded regions), scaling from SGR 1935's estimated age as $\tau_{\text {active }} \propto B^{-1.2}$ (Dall'Osso et al. 2012). The 5 orders of magnitude higher repetition rate of FRB 180916 would thus imply $B_{180916} \sim\left(10^{5}\right)^{1 / 3.2} B_{\text {SGR } 1935} \sim 10^{16} \mathrm{G}$, in agreement with separate lines of argument, e.g., requirements for FRB 180916 periodicity to be attributable to magnetar precession (Levin et al. 2020). We note that although the $\dot{E} \propto B^{3.2}$ scaling may seemingly be in tension with the larger inferred surface fields of SGR 1806-20 and SGR 1900+14 compared to SGR $1935+2154$, the relevant field in this scaling is instead the internal $B$ field, which is not directly observable (magnetar activity is also highly stochastic, and the implied $\dot{E}$ should be treated only in a time-averaged sense).

An alternative possibility is that the repetition rate increases significantly with the periodicity (Wadiasingh et al. 2020). In this case, the periodicity of 180916 (and its high activity relative to SGR 1935+2154) may be ascribed to an extremely long period magnetar (Beniamini et al. 2020). Interestingly, this option also requires a large internal magnetic field, $>10^{16} \mathrm{G}$, at birth (see Beniamini et al. 2020 for details).

The maximum distance up to which SGR 1935+2154's radio flare would be detectable by typical FRB search facilities sensitive to $F_{\text {lim }} \sim 1 \mathrm{Jy}$ ms fluence radio pulses is $D_{\text {lim }} \approx$ $12 \mathrm{Mpc} d_{10}\left(F_{\text {lim }} / 1 \mathrm{Jy} \mathrm{ms}\right)^{-1 / 2}$. The birth rate of ordinary Galactic magnetars in the local universe can be estimated as a fraction, $f_{\mathrm{CCSN}} \approx 0.1-1$ (Beniamini et al. 2019), of the $\mathrm{CCSN}$ rate, $\Gamma_{\mathrm{CCSN}} \approx(0.71 \pm 0.15) \times 10^{-4} \mathrm{Mpc}^{-3} \mathrm{yr}^{-1}(\mathrm{Li}$ et al. 2011). Thus, the rate of potentially detectable FRBs

\footnotetext{
12 Also note that activity may increase with the NS mass, because at sufficiently high central densities, the star can cool through direct URCA reactions, which hastens the release of magnetic energy from the core (Beloborodov \& Li 2016).
}

produced by SGR $1935+2154-$ like bursts is

$$
\begin{aligned}
\mathcal{R}\left(>F_{\text {lim }}\right) \approx & \frac{4 \pi}{3} D_{\text {lim }}^{3} f_{\mathrm{CCSN}} \Gamma_{\mathrm{CCSN}} \tau_{\text {active }} \lambda_{\text {mag }} \\
\in & {[0.004,1.4] \mathrm{sky}^{-1} \mathrm{day}^{-1} } \\
& \times d_{10}^{3}\left(\frac{f_{\mathrm{CCSN}}}{0.1}\right)\left(\frac{\tau_{\text {active }}}{10^{4} \mathrm{yr}}\right)\left(\frac{F_{\text {lim }}}{1 \mathrm{Jy} \mathrm{ms}}\right)^{-3 / 2} .
\end{aligned}
$$

This is much lower than the Parkes estimated FRB rate, $\approx 1.7_{-0.9}^{+1.5} \times 10^{3} \mathrm{sky}^{-1} \mathrm{day}^{-1}$, above a fluence of $2 \mathrm{Jy} \mathrm{ms}$ (Bhandari et al. 2018).

The estimate above shows that magnetars bursting at the same rate and energy as the April 28 SGR $1935+2154$ radio burst cannot noticeably contribute to the FRB population. However, magnetar flares clearly span a range of energies (Figure 1), making it natural to ask whether SGR 1935+2154like magnetars can reproduce the FRB rate if one extrapolates radio production with a similar efficiency to more energetic magnetar flares.

We therefore calculate the all-sky rate of FRBs above a limiting fluence threshold assuming that all magnetars repeat following a luminosity distribution $\lambda_{\operatorname{mag}}(>E) \propto\left(E / E_{\min }\right)^{-\gamma}$ for burst energies between $E_{\min }$ and $E_{\max }$. We set $E_{\max }=$ $\eta_{\text {mag }} E_{\text {mag }} \propto B^{2}$ dictated by the magnetic energy reservoir of the magnetar (this is consistent with the energies of the three observed Galactic giant flares relative to the magnetic dipole energies of the magnetars producing them; see, e.g., Tanaka et al. 2007) and fix the minimum energy to that observed for the April 28 SGR $1935+2154$ flare. $^{13}$ In the following, we assume $\eta_{\mathrm{mag}}=\eta \sim 10^{-5}$ (Equation (1)) is universal for all magnetar FRBs (motivated by the synchrotron maser model discussed in Section 5.2.2). The magnetar birth rate $\Gamma_{\text {birth }}(z)$ is assumed to follow the cosmic star formation rate (Hopkins \& Beacom 2006) and is anchored to $10 \%$ of the CCSN rate at $z=0$ (Li et al. 2011). The integrated rate is thus

$$
\begin{aligned}
\mathcal{R}\left(>F_{\text {lim }}\right)= & \int d V(z) \Gamma_{\text {birth }}(z) \tau_{\text {active }} \\
& \times \int d E \lambda_{\text {mag }}(E) \Theta\left[E-4 \pi D_{\mathrm{L}}(z)^{2} F_{\text {lim }}\right],
\end{aligned}
$$

where $d V(z)$ is the differential comoving volume element, $D_{\mathrm{L}}(z)$ is the luminosity distance, and $\Theta(x)$ is the Heaviside function.

We set $\tau_{\text {active }}=24 \mathrm{kyr}$ and fix $\lambda\left(>E_{\min }\right)$ to the estimated rate of SGR 1935 radio bursts. The former is determined from the minimum SNR age estimated by Zhou et al. (2020) and scaled to our adopted distance of $10 \mathrm{kpc}$. We then integrate over cosmological redshift and calculate the rate as a function of the slope of the luminosity function, $\gamma$. Figure 4 shows the resulting all-sky rate as a function of $\gamma$ (the only free variable in the calculation). For large $\gamma$, the rate is dominated by bursts of energy $\sim E_{\min }$, and we recover the result of Equation (3), namely, that the rate of Galactic magnetars is too low compared to cosmological FRBs. However, for $\gamma<1.5$, the rate becomes dominated by the high-energy tail of the luminosity function, and the number of detectable magnetar radio bursts increases. In particular, we find that, for $\gamma \lesssim 1$, magnetars of a similar kind to SGR $1935+2154$ can account for the entire FRB rate.

\footnotetext{
13 Given that the luminosity function we have adopted dictates fewer bursts at higher energies (for $\gamma>0$ ), it is statistically unlikely that the detected radio burst of SGR $1935+2154$ significantly exceeds $E_{\min }$.
} 


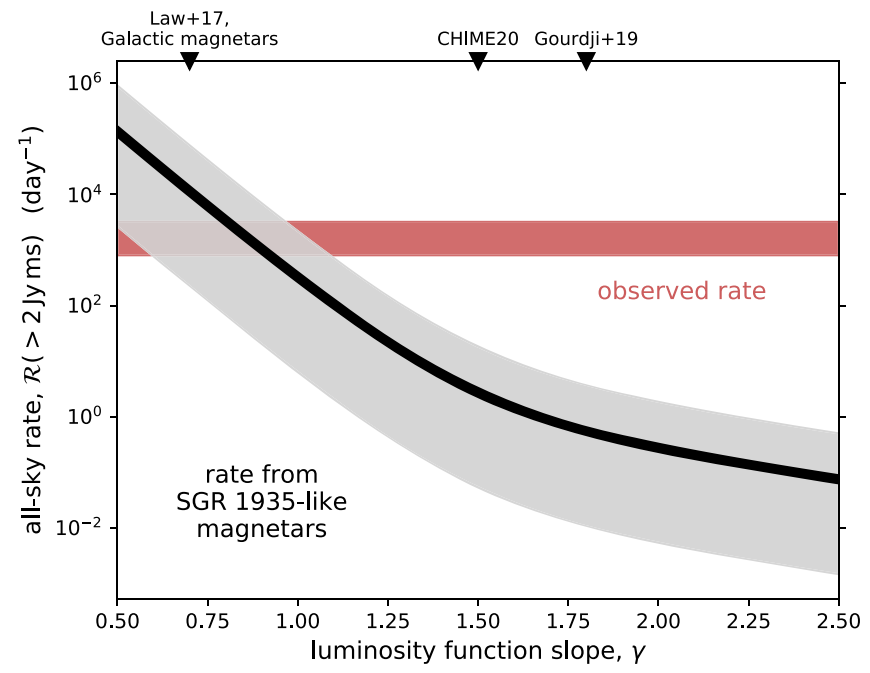

Figure 4. All-sky rate of FRBs above a limiting fluence of $2 \mathrm{Jy} \mathrm{ms}$. The black curve (and shaded uncertainty region) shows the FRB rate that would be detected from a single population of normal magnetars (assumed to be born at $10 \%$ of the CCSN rate) with active lifetimes and radio repetition rates fixed to that of SGR $1935+2154$ (equivalent to a fixed $B$ field). The repetition rate at $E_{\min }=2 \times 10^{34} \mathrm{erg}$ is fixed to the inferred rate of SGR $1935+2154$ bursts at this energy and extrapolated to higher energies by a luminosity function $\lambda_{\text {mag }}(>E) \propto E^{-\gamma}$. For large $\gamma$, the all-sky rate is dominated by weak, common bursts at $\sim E_{\min }$, and the rate falls short of the observed FRB rate (horizontal red; Bhandari et al. 2018) by several orders of magnitude (Equation (3)) However, for $\gamma \lesssim 1$, the rate of FRBs from SGR 1935+2154-like sources (extrapolated to higher energies) accommodates and even overproduces the observed FRB rate. The values of $\gamma$ found by different studies are indicated on the top axis, including the X-ray/gamma-ray luminosity function of Galactic magnetars.

We note that a low value of $\gamma \sim 0.7$ is consistent with estimates of Galactic magnetar X-ray burst fluences (e.g., Göğüś et al. 1999, 2000; Gavriil et al. 2004) and also potentially with FRB 121102 (Law et al. 2017; although see Gourdji et al. 2019). Separately, a value of $\gamma \sim 0.6$ has been suggested based on the slope count of nonlocalized ASKAP FRBs, with the assumption of a DM-distance relationship (Shannon et al. 2018; Lu \& Piro 2019).

Although extending the luminosity function of radio bursts to higher energies can allow (for certain values of $\gamma$ ) ordinary magnetars similar to SGR $1935+2154$ to reproduce the number of observed FRBs, this scenario falls short of explaining many other observables. In particular, and as discussed previously, the per-source activity rate for such a population would be far too low to explain prolific repeaters like FRBs 121102 and 180926. For the same reason, most FRB sources would be detected only once, and the relative number of repeaters versus apparently nonrepeaters would be very small. The scenario would also predict average FRB source distances that are much nearer than localized sources or DM-estimated distances.

\subsection{Two-component Population}

To further explore the requirements for possible magnetar progenitors of cosmological FRBs, we extend the calculation of the previous section and model a two-component magnetar population as necessitated by the observations: magnetars with low activity levels like SGR 1935+2154 and magnetars that are very active. A natural question this will allow us to address is whether the active population is consistent with an earlier evolutionary stage (a younger version) of the same SGR $1935+2154$-like population or whether one requires a distinct population altogether (e.g., a rare subset of magnetars born through unique channels).

We again calculate the all-sky FRB rate using Equation (4), accounting for the two populations contributing to FRB production. As before, the only free parameter describing the ordinary magnetar population is the luminosity function slope $\gamma$. The second, "active" population is then scaled from the former population as a function of the internal magnetic field $B$ and relative birth rate $f_{\mathrm{CCSN}}{ }^{14}$

The magnetic field of the magnetar enters in setting $E_{\max } \propto$ $B^{2}$, the active lifetime of sources $\tau_{\text {active }} \propto B^{-1.2}$ (Dall'Osso et al. 2012; Beloborodov \& $\mathrm{Li} 2016$ ), and the repetition rate $\lambda_{\text {mag }}\left(>E_{\text {min }}\right)$. The latter is proportional to $\propto B^{3.2}$ for $\gamma>1$ and $\propto B^{1.2+2 \gamma}$ for $\gamma<1 .{ }^{15} \mathrm{We}$ normalized $\tau_{\text {active }}$ and $\lambda_{\text {mag }}\left(E_{\text {min }}\right)$ at the magnetic field of SGR $1935+2154\left(\simeq 2.2 \times 10^{14} \mathrm{G}^{16}\right)$ to the age $\left(\gtrsim 24 d_{10} \mathrm{kyr}\right)$ and radio repetition rate of SGR 1935 . We then integrate Equation (4) and compare the resulting rate, a function of the three free parameters $\left\{B, \gamma, f_{\mathrm{CCSN}}\right\}$, to the observed FRB rate (Bhandari et al. 2018). From the point of view of the all-sky rate alone, there exists a degeneracy between the number of FRB sources in the universe $\left(\propto f_{\mathrm{CCSN}}\right)$ and the repetition rate of each source (a function of $B$ ). However, this degeneracy can be broken by constraining the observed repetition rate of prolific repeaters.

Figure 5 shows, for values of the magnetic field $B\left(\gamma, f_{\mathrm{CCSN}}\right)$ required to fit the observed FRB rate (and its uncertainties), the region in $\left\{\gamma, f_{\mathrm{CCSN}}\right\}$ parameter space where the average repetition rate of the active magnetar population equals the observed repetition rate of FRBs 121102 and 180916. For this, we take a fiducial value $\lambda_{\text {mag }}\left(>10^{38} \mathrm{erg}\right)=1 \mathrm{hr}^{-1}$ (see Figure 3 ); however, the uncertainties encompass a few orders of magnitude of leeway in this assumed value. On the basis of Figure 5, one can conclude that, regardless of the luminosity function slope $\gamma$, fitting both the all-sky FRB rate and the activity level of repeating FRBs requires a rare class of progenitors, $f_{\mathrm{CCSN}} \ll 1$. This is in line with many previous studies (e.g., Nicholl et al. 2017), and here we have extended these by adding the possibly inevitable (though likely subdominant) contribution of a second population of "normal magnetars" and utilizing scaling relations anchored to the new observations of SGR $1935+2154$.

The fact that $f_{\mathrm{CCSN}} \ll 1$ is required of the active population implies that these sources cannot be interpreted as younger incarnations of the SGR 1935+2154-like magnetar population as a whole, since this would require the same birth rate for both populations, i.e., $f_{\mathrm{CCSN}} \sim 0.1$. In the above, we have assumed isotropic radio emission, as we expect beaming to be modest at most (Section 4). If radio emission is significantly beamed, then the number of FRB-emitting magnetars in the universe would be larger.

To further compare the resulting population against observational constraints, we calculate the expected number of repeating and apparently nonrepeating FRB sources detected by a mock survey of this population. We assume a limiting fluence of $F_{\text {lim }}=4 \mathrm{Jy} \mathrm{ms}$ and average repeat-field exposure

\footnotetext{
14 We assume that the ordinary magnetar population is born at $10 \%$ of the CCSN rate, independent of the fractional birth rate $f_{\mathrm{CCSN}}$ of the active population.

${ }^{15}$ This is calculated based on the magnetic field scaling of $E_{\max }$ and $E_{\min }$, the fact that $\dot{E} \propto \lambda_{\operatorname{mag}}\left(>E_{\min }\right) E_{\min }$ for $\gamma>1$ and $\dot{E} \propto \lambda_{\operatorname{mag}}\left(>E_{\max }\right) E_{\max }$ for $\gamma>1$, and that $\dot{E} \propto B^{3.2}$ (Dall'Osso et al. 2012; Beloborodov \& Li 2016).

16 This value is based on the inferred external dipole field of SGR $1935+2154$. The internal field may, in fact, be larger.
} 


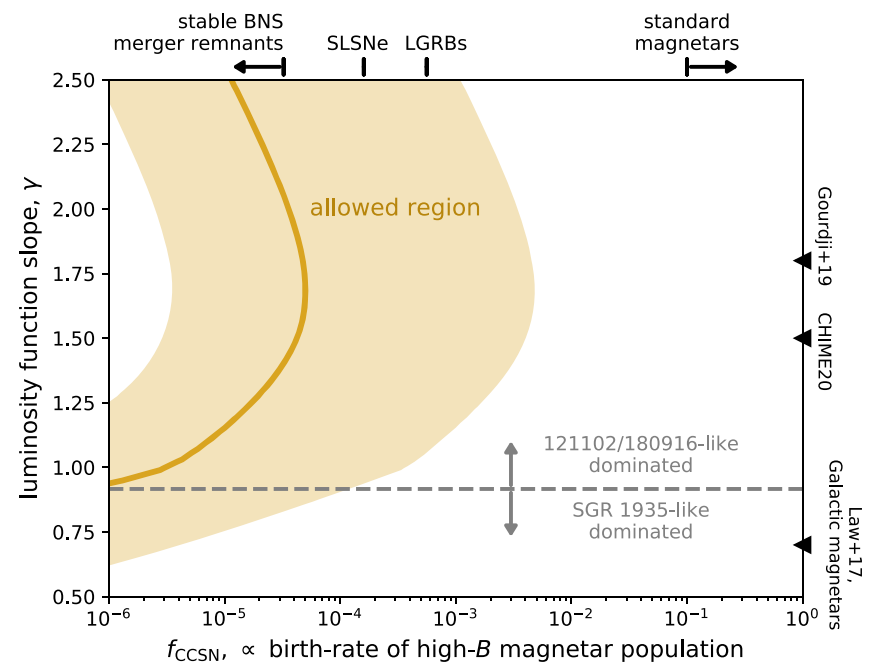

Figure 5. Two-component magnetar population consisting of (i) ordinary magnetars fixed to properties of SGR $1935+2154$ and extrapolated as a function of the luminosity function slope $\gamma$ and (ii) a magnetar population whose activity rate (parameterized via the internal magnetic field) and birth rate (a fraction $f_{\mathrm{CCSN}}$ of the CCSN rate) are allowed to vary (the value of $\gamma$ is identical to both populations). The yellow curve and shaded uncertainty region show the allowed parameter space constrained by both the all-sky FRB rate and the per-source activity (repetition rate). The latter constraint forces $f_{\mathrm{CCSN}} \ll 1$, indicating that the second, active population of magnetars must be volumetrically rare. This rules out the hypothesis that active cosmological repeating FRBs are younger versions of all SGR 1935+2154-like magnetars (for which $f_{\mathrm{CCSN}} \gtrsim 0.1$ ).

time of $T_{\exp }=40 \mathrm{hr}$, parameters motivated by the CHIME FRB survey. We then calculate the (Poissonian) number of sources for which only a single burst would be detected,

$$
N_{\text {nonrep }}=\int d V(z) f_{\text {mag }} \Gamma_{\text {birth }}(z) \tau_{\text {active }} \mu e^{-\mu},
$$

where

$$
\mu(z) \equiv \lambda_{\mathrm{mag}}\left(>4 \pi D_{\mathrm{L}}(z)^{2} F_{\text {lim }}\right) T_{\mathrm{exp}},
$$

and summing the contribution from both active and SGR 1935 +2154-like populations. The number of sources classified as repeaters by the same survey is similarly calculated as

$$
N_{\text {rep }}=\int d V(z) f_{\text {mag }} \Gamma_{\text {birth }}(z) \tau_{\text {active }}\left[1-\frac{1}{2} \Gamma(2, \mu)\right] \text {, }
$$

where $\Gamma(2, \mu)$ is the incomplete gamma function.

Figure 6 shows $N_{\text {rep }}$ and $N_{\text {nonrep }}$ as a function of $\gamma$ and for a representative value of $f_{\mathrm{CCSN}}=10^{-4}$ that is consistent with the constraints on FRB 121102-like activity and the all-sky FRB rate (Figure 5). The number of detected repeating and nonrepeating sources can be compared to values from the CHIME FRB survey, shown as horizontal curves (V. Kaspi 2020, private communication). The figure shows that, for values $1 \lesssim \gamma \lesssim 1.5$, the absolute number and relative ratio of repeating and nonrepeating FRBs can be reproduced simultaneously with the all-sky rate and per-source activity rate. For these values of $\gamma$, the rates of both repeaters and apparent nonrepeaters are dominated by the active population. At low values of $\gamma$, the observed FRBs are dominated by the less active ordinary magnetar population. This results in a significant reduction in the relative number of repeating versus nonrepeating sources that is inconsistent with observations. This substantiates our previous claim that a single population of (or, equivalently, a population dominated by) SGR 1935 +2154-like magnetars cannot account for the number of known repeaters.

Finally, using the cumulative distribution of detected events implied by Equations (5) and (7), we calculate the characteristic distances at which repeating and nonrepeating FRBs would be detected by this mock survey. The right panel of Figure 6 shows the median distance of detected repeating and apparently nonrepeating FRB sources as a function of $\gamma$. Confirmed repeaters are detected, on average, at a lower distance, broadly consistent with the $149 \mathrm{Mpc}$ distance of the first localized CHIME repeater (and note that FRB 121102, at a much larger distance of $972 \mathrm{Mpc}$, is detected only once by CHIME, consistent with the median distance of apparently nonrepeating sources detected by the mock survey).

As the model shows, many potential rare magnetar formation channels could, in principle, be consistent with the all-sky FRB and repeater rate constraints (Figure 5). One way to further break this degeneracy is via host galaxy demographics. A high- $B$ (and hence potentially particularly slowly rotating; Beniamini et al. 2020) tail of the magnetar population should be formed in otherwise ordinary CCSNe and hence track star-forming galaxies almost exclusively. Superluminous supernovae (SLSNe) and long-duration GRBs (LGRBs) should originate predominantly (Fruchter et al. 2006; Lunnan et al. 2015; Blanchard et al. 2016), though not exclusively (e.g., Perley et al. 2017), in dwarf starforming galaxies. By comparison, NS mergers, white dwarf-NS mergers, and accretion-induced collapse (AIC) should originate from a range of star-forming and non-star-forming galaxies (Margalit et al. 2019), weighted more toward massive galaxies than SLSNe/LGRBs. Attempts to perform an analysis along these lines are already underway (e.g., Margalit et al. 2019; Li \& Zhang 2020), though it should be cautioned that without at least arcsecond localization, it is usually challenging to uniquely identify the host galaxy (Eftekhari et al. 2018), much less the local environment within the host galaxy, as it becomes available with VLBI localization (Bassa et al. 2017; Marcote et al. 2020).

\section{Implications for Magnetar FRB Models}

A clear link between FRBs and magnetars is provided by SGR 1935+2154. Such a connection was proposed and discussed extensively in the FRB literature well prior to this discovery (e.g., Popov \& Postnov 2013; Kulkarni et al. 2014; Lyubarsky 2014; Katz 2016; Beloborodov 2017; Kumar et al. 2017; Metzger et al. 2017), leading to the development of several distinct magnetar models for FRBs. Broadly speaking, these models can be further divided based on whether the radio emission originates from near the NS magnetosphere (the "curvature," "low-twist," and "reconnection" models) or at much further distances ("synchrotron maser blast wave" models). We also briefly discuss a couple of NS-related models not specific to magnetars. In the following, we discuss the implications of the SGR $1935+2154$ radio burst for these models, pointing out the strengths and points of contention between each and the combined radio and X-ray observations.

\subsection{Low-twist Models}

In the low-twist models (Wadiasingh \& Timokhin 2019; Wadiasingh et al. 2020), magnetic field dislocations and oscillations in the NS surface can lead to pair cascades, assuming that the background charge density is sufficiently 

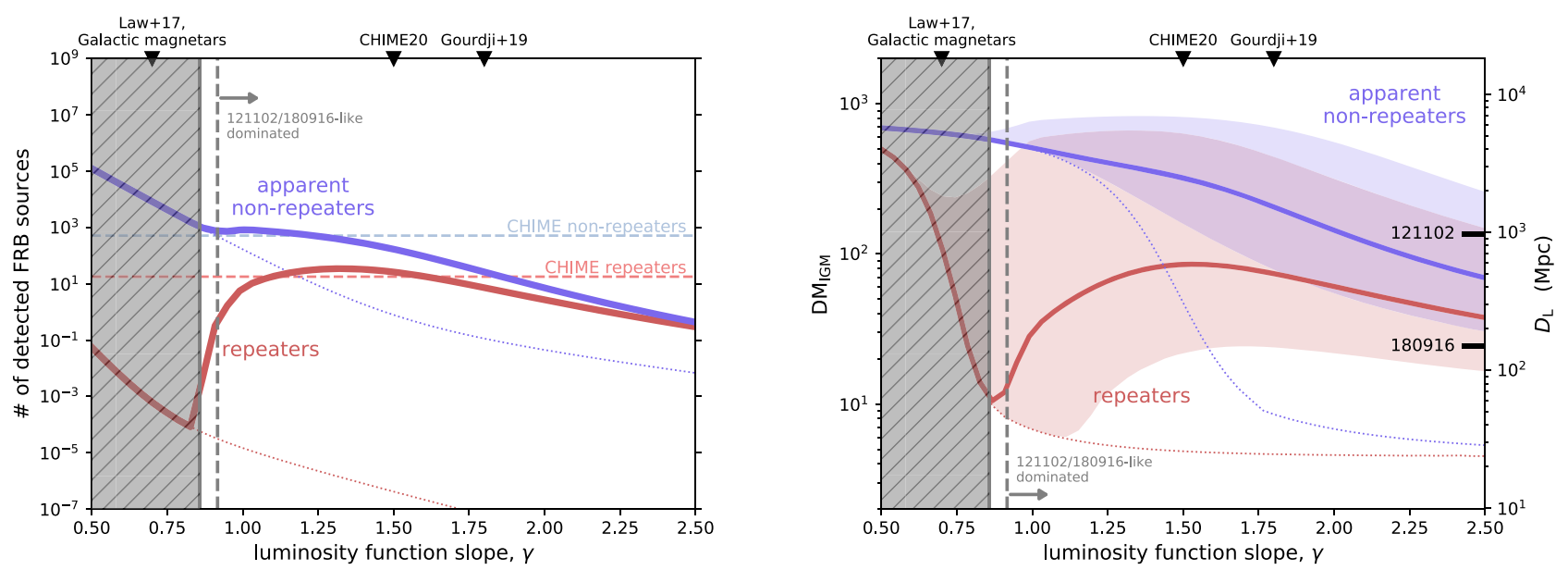

Figure 6. Several properties of cosmological FRBs can be simultaneously reproduced by postulating the existence of a rare population of magnetars (with a birth rate taken to be $f_{\mathrm{CCSN}}=10^{-4}$ of the CCSN rate in this example) with stronger magnetic fields than the Galactic magnetar population. Left: for a mock survey with parameters motivated by the CHIME/FRB experiment, the number of detected repeating sources (solid red) and apparently nonrepeating sources (solid blue) as a function of the power-law slope $\gamma$ of the FRB luminosity function $\lambda(>E) \propto E^{-\gamma}$. The numbers of repeating and nonrepeating sources actually detected by CHIME are shown as light blue and red dashed horizontal curves, respectively (V. Kaspi 2020, private communication). The thin dotted curves show the contribution of the ordinary magnetar population to the number of detected sources. This population is subdominant for $\gamma \gtrsim 1$ and dominant at low $\gamma$, where the all-sky FRB rate is overproduced (hatched gray region; see also Figure 4). Right: median distance (right axis) and corresponding DM contribution from the intergalactic medium (left axis) of sources detected as repeaters (solid red) and nonrepeaters (solid blue) for the same mock survey. The distances of FRB 180916 and FRB 121102 (which, from CHIME's point of view, is a nonrepeater) are shown for comparison. For clarity, uncertainties associated with the all-sky FRB rate, the repetition rate of SGR 1935 +2154 , etc. are not shown in these plots.

low. The latter then result in coherent radio emission. Since it is the same dislocation that supposedly results in short X-ray bursts and (in some cases) FRBs, a prediction of this model is that all FRBs should be associated with short magnetar bursts (but not vice versa). This is consistent with the observations of SGR 1935. Another attractive feature of this model is that FRBs will typically be associated with older magnetars, consistent with the putative age of SGR 1935. However, the association with older ages in the model is due to the requirement of longer periods, while the period of SGR 1935 +2154 is very typical as compared to other Galactic magnetars. Similarly, the model favors strong dipole field strengths, while the dipole field of SGR 1935+2154 does not appear to be exceptional relative to other magnetars.

In the low-twist model, both the radio emission and the $\mathrm{X}$-rays arrive from the magnetosphere. As such, this model predicts a radius-to-frequency mapping leading to frequency selection, similar to the case in pulsars and potentially a polarization angle that is varying with time (as the magnetic field orientation changes relative to the observer). The X-ray burst associated with an FRB should exhibit a standard blackbody (or double blackbody) spectrum as seen in other short X-ray bursts.

\subsection{Synchrotron Maser Blast-wave Models}

In synchrotron maser models, a version of which was first proposed by Lyubarsky (2014), FRBs are created via the coherent synchrotron maser process that is naturally produced in magnetized relativistic shocks (Gallant et al. 1992; Plotnikov \& Sironi 2019). Such shocks are expected to arise from relativistic flares that may be ejected during magnetar outbursts. A number of variants on the synchrotron maser model exist that differ regarding the nature of the upstream medium and the required shock properties; however, in all cases, the bursts are powered by tapping into a small fraction of the kinetic energy of the outflow and predict corresponding (though differing) high-frequency counterparts to FRBs.

\subsubsection{Magnetar Wind Nebula (Lyubarsky 2014)}

Lyubarsky (2014) proposed that FRB production occurs as the ultrarelativistic flare ejecta collides with the pulsar wind nebula. The radius of the termination shock is estimated to be

$$
r_{\mathrm{s}}=\sqrt{\frac{L_{\mathrm{sd}}}{4 \pi p c}}
$$

where $p$ is the pressure inside the nebula. Taking $L_{\mathrm{sd}} \simeq 1.7 \times$ $10^{34} \mathrm{erg} \mathrm{s}^{-1}$ and $p \sim 10^{-9} \mathrm{erg} \mathrm{cm}^{-3}$ (estimated from the energy of $10^{51}$ erg of a typical supernova and the observed $\sim 20 \mathrm{pc}$ size of the SNR surrounding SGR 1935+2154; Kothes et al. 2018), we find $r_{\mathrm{s}} \approx 6 \times 10^{15} \mathrm{~cm}$. The light-crossing timescale to this radius is $r_{\mathrm{s}} / c \sim 2$ days. However, because the flare ejecta and the resulting shock are also moving close to the speed of light, radio photons from the shocked nebula could, in principle, still arise nearly simultaneously with the X-rays, which in this model presumably must be generated from the inner magnetosphere.$^{17}$ If the X-ray photons are emitted within the magnetosphere concurrently with the relativistic flare ejection, the X-rays would be expected to arrive a short time, $\sim r_{\mathrm{s}} / 2 \Gamma^{2} c \sim \mathrm{ms}$, before the radio, which is potentially in tension with the timing estimated by Mereghetti et al. (2020a). However, we note that various uncertainties in this timing may affect this conclusion (see Section 5.2.2 for further details).

Looking more closely at the predictions for the radio emission requires rescaling the results of Lyubarsky (2014),

\footnotetext{
17 Although a burst of higher-frequency (incoherent) synchrotron is predicted in this model from the shocked electrons, for parameters appropriate to SGR $1935+2154$, this is predicted to occur at $\sim 20 \mathrm{GeV}$ gamma-rays with a fluence of $\sim 10^{-5} \mathrm{erg} \mathrm{cm}^{-2}$.
} 
who considered a giant flare that carries away a significant fraction of the magnetic energy of the star. The recent flare from SGR 1935+2154 was less energetic by a factor of $\sim 10^{6}$, corresponding to a strength of the magnetic field in the pulse smaller by a factor of $\sim 10^{3}$, i.e., dimensionless constant $b \sim 10^{-3}$ in the notation of Lyubarsky (2014). Following Equation (8) of Lyubarsky (2014) for these parameters, the predicted peak frequency of the maser emission from the forward shock is estimated to be $\nu_{\mathrm{pk}} \sim 10 \mathrm{MHz}$. Given the drop-off in the $\nu L_{\nu}$ spectrum of the maser from $\nu_{\mathrm{pk}}$ to $\sim 100 \nu_{\mathrm{pk}}$ by a factor of $\sim 10^{-4}$ (Plotnikov \& Sironi 2019), the fraction of the total radio emission in the $1.4 \mathrm{GHz}$ band of STARE2 would be only $\sim 10^{-4}$. Given an intrinsic efficiency of the synchrotron maser emission of $f_{\xi} \sim 10^{-2}-10^{-3}$ (see next section), the resulting net efficiency of the radio emission of $\sim 10^{-6}-10^{-7}$ is at best marginally consistent with the observations if the energy of the flare ejecta were comparable to the released X-ray fluence. The fact that the second radio burst detected by CHIME appears to have a rising spectrum is also in tension with this model (since $\nu_{\mathrm{pk}} \sim 10 \mathrm{MHz}$, as discussed above), although scintillation may affect the observed spectrum (Simard \& Ravi 2020).

\subsubsection{Baryonic Shell (Metzger et al. 2019; Margalit et al. 2020)}

In this version of the synchrotron maser model, first proposed by Beloborodov (2017), the ultrarelativistic head of the magnetar flare collides not with the magnetar wind nebula but rather with matter ejected from a recent, earlier flare. Motivated by the inference from the radio afterglow of the 2004 giant flare from SGR 1806-20 of a slow ejecta shell generated by the burst (Gelfand et al. 2005; Granot et al. 2006), Metzger et al. (2019) considered the upstream medium to be a subrelativistic baryon-loaded shell with an electron-ion composition.

The low efficiency of radio emission implied that the X-ray and radio observations of SGR 1935+2154 (Equation (1)) are consistent with the predictions of this model. The radio inefficiency is attributable to a combination of the intrinsic synchrotron maser efficiency $f_{\xi} \sim 10^{-2}-10^{-3}$ (for moderate magnetization; Plotnikov \& Sironi 2019) and a further reduction by a factor of $\sim 10^{-2}$ due to the effects of induced Compton scattering suppressing the low-frequency portion of the maser's intrinsic SED (see Metzger et al. 2019, their Section 3.2). ${ }^{18}$

Following the methodology of Margalit et al. (2020), we can use the energy, frequency, and duration of the observed radio burst to derive the intrinsic parameters of the flare demanded by the synchrotron maser model. Adopting the observed quantities from Section 2, we find that the energy of the relativistic flare, $E_{\text {flare }}$; the Lorentz factor $\Gamma$ of the shocked gas at the time the observed radio flux is emitted; the radius of the shock from the central magnetar at this time, $r_{\mathrm{sh}}$; and the external density of the

\footnotetext{
18 The value of $f_{\xi}$ in general decreases with increasing values of the upstream magnetization, $\sigma$. Based on 1D particle-in-cell simulations of electron-positron plasmas, Plotnikov \& Sironi (2019) found an efficiency of

$$
f_{\xi}=7 \times 10^{-4} / \sigma^{2}, \sigma \gg 1 \text {. }
$$

Matching $f_{\xi}=\eta \sim 10^{-5}$ (Equation (1)) places a strict upper limit of $\sigma \lesssim 8$. The true efficiency (and hence allowed $\sigma$ ) will be lower once accounting for 3D effects, electron-ion composition of the upstream plasma (e.g., Iwamoto et al. 2019), and suppression of the radio signal from induced scattering by upstream electrons (Metzger et al. 2019). This scenario therefore requires an upstream plasma that is not highly magnetized.
}

upstream baryonic shell at this location, $n_{\mathrm{ext}}$, are given by

$$
\begin{gathered}
E_{\text {flare }} \approx 7 \times 10^{39} \operatorname{erg} f_{\xi,-3}^{-4 / 5} d_{10}^{2}, \\
\Gamma \approx 56 f_{\xi,-3}^{-1 / 15} d_{10}^{1 / 3}, \\
n_{\mathrm{ext}} \approx 4 \times 10^{4} \mathrm{~cm}^{-3} f_{\xi,-3}^{-4 / 15} d_{10}^{-2 / 3}, \\
r_{\mathrm{sh}} \approx 1.1 \times 10^{11} \mathrm{~cm} f_{\xi,-3}^{-2 / 15} d_{10}^{2 / 3} .
\end{gathered}
$$

In the above, we adopt the STARE2 fluence and burst duration (Bochenek et al. 2020b) and express the results as a function of the maser efficiency $f_{\xi}=10^{-3} f_{\xi,-3}$.

From our inferred parameters, the (very) local DM contributed by the immediate upstream medium ahead of the shock is $\mathrm{DM} \gtrsim n_{\mathrm{ext}} r_{\mathrm{sh}} \approx 10^{-3} \mathrm{pc} \mathrm{cm}^{-3}$ and can exceed this value significantly if the upstream medium extends to larger radii. In the context of the shock model, it may be expected that DM variations could exist between radio bursts on this order of magnitude or larger. Note, however, that nonlinear wave interaction with the upstream plasma, as well as strong upstream magnetic fields, may inhibit the DM of this local environment (e.g., Lu \& Phinney 2020).

The derived shock properties are shown in Figure 7 in comparison to those derived for cosmological FRBs within the same model for an assumed efficiency $f_{\xi}=10^{-3}$. One important thing to note is that the flare energy $E_{\text {flare }}$ that the model demands (based on the radio observation of SGR 1935 +2154 alone) agrees remarkably well with the independently observed X-ray energy, $E_{\mathrm{X}} \approx 8 \times 10^{39} \mathrm{erg}$. As we discuss below, such an agreement is naturally expected if electrons heated at the shock generate the X-rays via synchrotron radiation in the fast-cooling regime.

Stated more directly, the model predicts a ratio (Margalit et al. 2019),

$$
\begin{aligned}
\eta_{\text {theory }} & \equiv \frac{E_{\text {radio }}}{E_{\text {flare }}}=\left(\frac{1215}{512 \pi^{2}} \frac{m_{\mathrm{e}}}{m_{\mathrm{p}}}\right)^{1 / 5} f_{\mathrm{e}}^{1 / 5} f_{\xi}^{4 / 5}\left(\nu_{\mathrm{obs}} \cdot t_{\mathrm{FRB}}\right)^{-1 / 5} \\
& \sim 3 \times 10^{-5}\left(\frac{f_{\mathrm{e}}}{0.5}\right)^{1 / 5} f_{\xi,-3}^{4 / 5}\left(\frac{\nu_{\mathrm{obs}} \cdot t_{\mathrm{FRB}}}{1 \mathrm{GHz} \cdot \mathrm{ms}}\right)^{-1 / 5},
\end{aligned}
$$

that matches the observed ratio, $\eta \equiv E_{\text {radio }} / E_{\mathrm{X}} \sim 10^{-5}$ (Equation (1)), for the expected values of the maser efficiency $f_{\xi} \sim 10^{-3}-10^{-2}$ (Plotnikov \& Sironi 2019), provided that $E_{\mathrm{X}} \sim E_{\text {flare. }}$ In the above, $f_{\mathrm{e}}$ is the ratio of electron to ion number densities in the upstream medium, and $f_{\mathrm{e}} \sim 1$ for an electron-ion plasma we consider.

The same outwardly propagating shock that generates the coherent precursor radio emission (the FRB) in this scenario also generates incoherent synchrotron radiation from relativistically hot electrons heated by the shock (Lyubarsky 2014; Metzger et al. 2019), somewhat akin to a GRB afterglow. Using the shock parameters implied by the radio observations (Figure 7), we now assess the predicted properties of the highfrequency counterpart, showing it to be in accord with the X-ray emission from SGR $1935+2154$.

The peak frequency of the "afterglow" is set by the characteristic synchrotron frequency, which, for an ultrarelativistic blast wave decelerating into an effectively stationary upstream medium of magnetization $\sigma=10^{-1} \sigma_{-1}$, is given by 


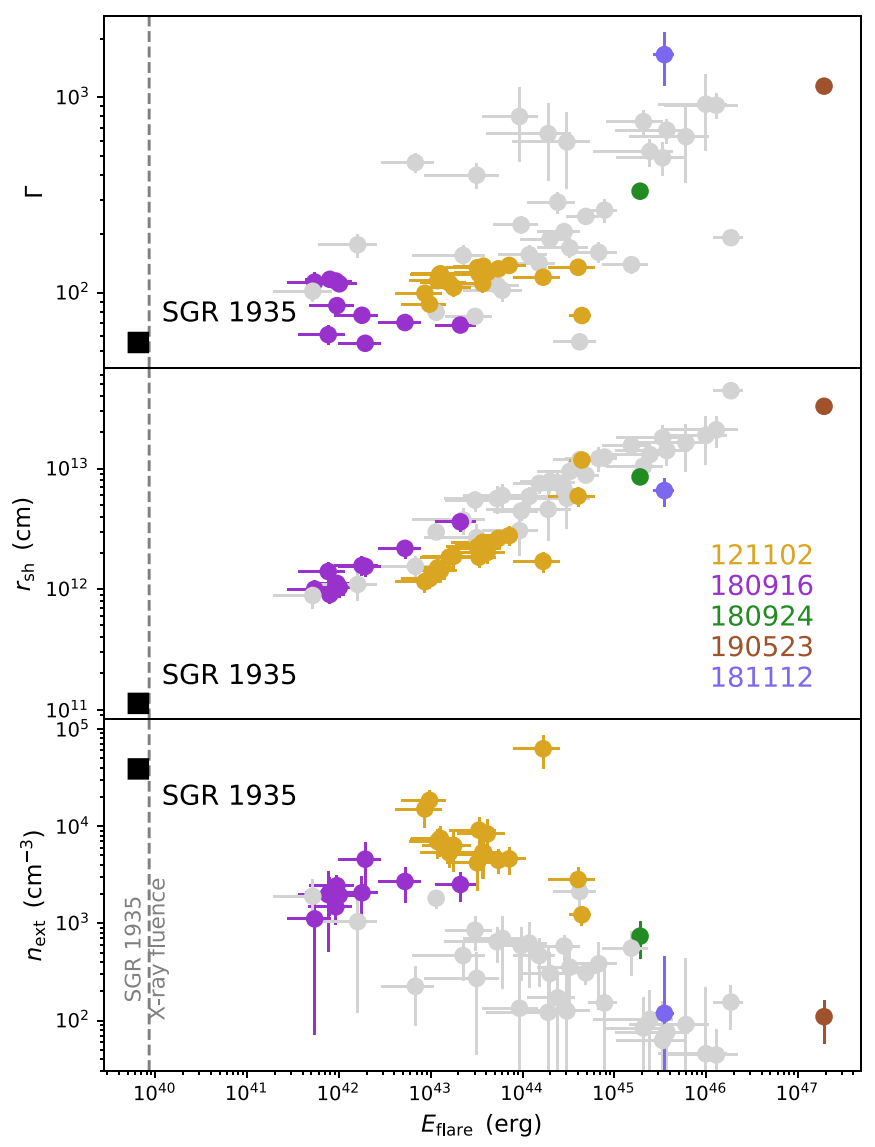

Figure 7. Derived properties from the SGR 1935 radio burst within the variation of the synchrotron maser model in which the upstream medium is a baryon-loaded shell and adopting a fiducial value for the maser efficiency $f_{\xi}=10^{-3}$ (see also Margalit et al. 2020). From top to bottom, we show the bulk Lorentz factor $\Gamma$, radius $r_{\mathrm{sh}}$, and external upstream density $n_{\mathrm{ext}}$ of the shock at the time of the observed radio flare, all as a function of the derived total flare energy, $E_{\text {flare }}$. The flare energy, as derived from radio observations alone, is comparable to the detected X-ray counterpart of this burst (vertical dashed curve), in line with the predictions of the synchrotron maser model (Section 5.2.2) if the X-rays arise from thermal synchrotron radiation from the same shocks that generate the FRB.

(Metzger et al. 2019; their Equations (56) and (57))

$$
\begin{aligned}
E_{\text {peak }} & \sim h \nu_{\text {syn }} \approx 110 \mathrm{keV}\left(\frac{E_{\text {flare }}}{10^{39} \mathrm{erg}}\right)^{1 / 2} \sigma_{-1}^{1 / 2}\left(\frac{t}{3 \mathrm{~ms}}\right)^{-3 / 2} \\
& \approx 290 \mathrm{keV} \sigma_{-1}^{1 / 2}\left(\frac{t}{3 \mathrm{~ms}}\right)^{-3 / 2} f_{\xi,-3}^{-2 / 5} d_{10}
\end{aligned}
$$

where $t$ is the time since the peak of the flare, and we have assumed that half of the kinetic power dissipated at the shock goes into heating electrons into a relativistic Maxwellian distribution (supported, e.g., by particle-in-cell simulations of magnetized shocks; Sironi \& Spitkovsky 2011). In the second line of Equation (15), we have substituted the flare energy from Equation (13) needed to reproduce the radio burst properties from SGR 1935+2154, and we take the X-ray burst duration to be $\sim 3 \mathrm{~ms}$, consistent with observations (Li et al. 2020). Although the value of $\sigma$ in the flare ejecta of a magnetar flare is uncertain theoretically, there is only a small dynamical range it can attain if this model is to explain FRB radio observations: a minimum magnetization $\sigma \gtrsim 10^{-3}$ is required for the synchrotron maser to operate in the first place, while the declining efficiency of the maser emission with increasing $\sigma$ places an upper limit of $\sigma \lesssim 1$.

For the same parameters, the cooling frequency is given by

$$
\begin{aligned}
h \nu_{\mathrm{c}} & \approx 1.4 \mathrm{keV} \sigma_{-1}^{-3 / 2}\left(\frac{n_{\mathrm{ext}}}{10^{4} \mathrm{~cm}^{-3}}\right)^{-3 / 2}\left(\frac{\Gamma}{100}\right)^{-4}\left(\frac{t}{3 \mathrm{~ms}}\right)^{-2} \\
& \approx 22 \mathrm{keV} \sigma_{-1}^{-3 / 2}\left(\frac{t}{3 \mathrm{~ms}}\right)^{-1 / 2} f_{\xi,-3}^{2 / 3} d_{10}^{-1 / 3}
\end{aligned}
$$

where the particular temporal scaling $\propto t^{-1 / 2}$ is derived assuming a radially constant density profile $\left(n_{\text {ext }} \propto r^{0}\right)$.

The fact that $\nu_{\mathrm{c}} \lesssim \nu_{\text {syn }}$ on the timescales $t \sim 3 \mathrm{~ms}$ of interest shows that the postshock electrons are fast-cooling, and hence a large fraction of the flare energy, $E_{\text {flare, }}$ is emitted as hard $\mathrm{X}$-rays of energy $E_{\text {peak }} \sim 10-100 \mathrm{keV}$. The predicted X-ray spectrum is thus fast-cooling synchrotron emission from relativistically hot electrons with a thermal Maxwellian energy distribution (see Giannios \& Spitkovsky 2009, their Figure 3), resulting in an ordinary fast-cooling spectrum $\nu L_{\nu} \propto \nu^{1 / 2}$ between $\nu_{\mathrm{c}}$ and $\nu_{\mathrm{syn}}$ and an exponential cutoff at an energy $\sim \nu_{\text {syn }}$. This is broadly consistent with the observed photon index of $\sim 1.5$ inferred by HXMT for the SGR $1935+2154 \mathrm{X}$ ray counterpart ( $\mathrm{Li}$ et al. 2020). Indeed, modeling of other bright magnetar flares suggests that they can be well fit by a cutoff power-law spectrum in the X-rays (van der Horst et al. 2012).

Extending the same model to the shock properties derived for the observed populations of cosmological FRBs predicts that the afterglow emission for these more energetic bursts will occur at much higher energies, $E_{\text {peak }} \gtrsim \mathrm{MeV}-\mathrm{GeV}$, in the gamma-ray band (Figure 8). Unfortunately, gamma-ray satellites like Swift and Fermi are generally not sensitive enough to detect this emission to the cosmological distances of most FRB sources (Metzger et al. 2019; Chen et al. 2020; Margalit et al. 2020). We furthermore emphasize that this predicted short $(\sim$ milliseconds duration) gamma-ray signal from the shocks is distinct from the longer-lasting and typically softer gamma-ray emission observed from giant Galactic magnetar flares (e.g., Hurley et al. 2005; Palmer et al. 2005), which is instead well explained as a pair fireball generated by dissipation very close to the NS surface. The latter being relatively isotropic compared to the relativistically beamed radio emission from the ultrarelativistic shocks (hypothesized to accompany the beginning of the flare; Lyubarsky 2014; Beloborodov 2017) might explain the nondetection of FRB-like emission from the 2004 giant flare of SGR 1806-20 (Tendulkar et al. 2016).

If the X-rays from magnetar flares are attributable to thermal synchrotron shock emission, this may be imprinted in correlations between X-ray observables. Since the electrons behind the shock are fast-cooling, the X-ray fluence $F_{\mathrm{X}}$ should scale linearly with the flare energy $E_{\text {flare}}$, from which one predicts from Equation (15) a correlation (for fixed $\sigma$ )

$$
E_{\text {peak }} \propto F_{\mathrm{X}}^{1 / 2} t_{\mathrm{X}}^{-3 / 2}
$$

between the spectral energy peak, burst fluence, and some measure of the burst duration $t_{\mathrm{X}}$.

For X-ray bursts from the Galactic magnetar SGR J1550-5418, van der Horst et al. (2012) reported a correlation between the fluence and "emission" time, $\tau_{90} \propto F_{\mathrm{X}}^{0.47}$. Taking 


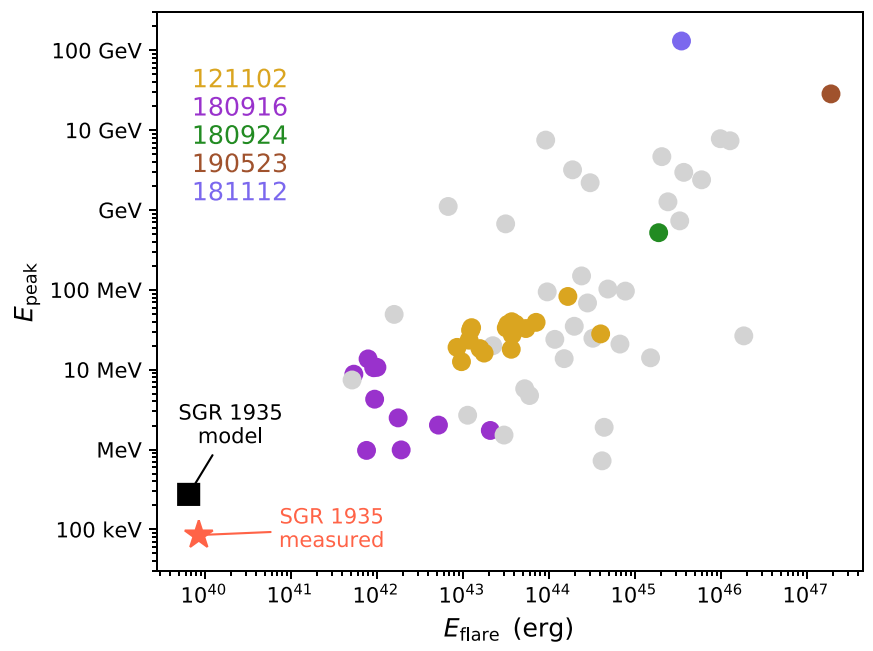

Figure 8. The synchrotron maser model predicts that FRBs are accompanied by hard radiation counterparts, the properties of which can be derived by modeling the observed radio emission alone. Above, we show the peak energy of this counterpart as a function of flare energy, derived for a sample of cosmological FRBs and the SGR 1935+2154 radio burst, assuming a uniform magnetization $\sigma=0.1$ for the upstream medium. The emitting electrons are fast-cooling; therefore, $E_{\text {flare }}$ roughly corresponds to the radiated fluence at $E_{\text {peak }}$. The observed $E_{\text {peak }}$ and fluence of the contemporaneous X-ray burst associated with SGR $1935+2154$ 's radio emission agree remarkably well with the model prediction.

$t_{\mathrm{X}} \propto \tau_{90}$, Equation (17) predicts $E_{\text {peak }} \propto F_{X}^{-0.2}$, which is close to but slightly shallower than the correlation $E_{\text {peak }} \propto F_{X}^{-0.44}$ found by van der Horst et al. (2012) using the entire burst sample. However, note that the most energetic bursts studied by van der Horst et al. (2012) exhibit a flatter or even positive correlation of $E_{\text {peak }}$ with fluence. A correlation very close to $E_{\text {peak }} \propto F_{X}^{-0.44}$ is also predicted from Equation (17) using the empirical relationship $F_{\mathrm{X}} \propto t_{\mathrm{X}}^{1.54}$ found between the bursts from different magnetars (Gavriil et al. 2004; see Figure 1). Thus, we advance the hypothesis that hard X-ray emission in even ordinary magnetar flares is generated by internal shocks in baryon-loaded outflows.

Scaling the shock model to the lower values of $r_{\mathrm{sh}}, \Gamma$ than derived for SGR $1935+2154$ above would also have potential consequences for the X-ray spectrum. In particular, the synchrotron spectrum could become opaque to pair creation in some X-ray counterparts of Galactic magnetar flares. Following Lithwick \& Sari (2001) and Beniamini \& Giannios (2017), we calculate the pair creation opacity corresponding to the model parameters described above and find the external shock to be optically thin to pair creation. Pair creation could, however, become important for somewhat lower values of $r_{\mathrm{sh}}, \Gamma$, which could be realized if the dissipation is due to internal shocks at a radius that is much lower than $r_{\mathrm{sh}}$. For example, keeping $E_{\text {flare }}$ fixed and reducing the Lorentz factor to $\Gamma \sim 4$ will lead to a pair creation cutoff at the internal shock site at $\sim 40 \mathrm{keV}$. We speculate that this might explain why the high temperature of X-ray bursts described by a double blackbody spectrum typically peaks at energies $\lesssim 40 \mathrm{keV}$.

Finally, the explanation provided here for the X-ray spectra of Galactic magnetar short bursts might apply most robustly only to the smaller subset of bright SGR bursts that are luminous enough to drive radiation-driven outflows (i.e., large values of $L_{\mathrm{X}} / L_{\text {min }}$; see Figure 2). In this regard, it is interesting that the brightest bursts analyzed by van der Horst et al. (2012) exhibit a different correlation between their peak energy and flux to the rest of the bursts, consistent with the idea that the physical mechanism driving these bursts is different.

Returning to the radio burst emission, Metzger et al. (2019) predicted a downward drift in frequency ${ }^{19}$ as the shock decelerates and the (Lorentz boosted) synchrotron maser emission sweeps from high to low frequencies. The presence (or lack) of this feature in the SGR 1935+2154 burst would therefore provide a helpful diagnostic of FRB models and a probe of the density profile in this specific burst (see Margalit et al. 2020 for application to CHIME repeaters). In addition to the in-band drift that may potentially be detectable by CHIME (although the nontrivial frequency response of the CHIME side lobes may hinder this), the same process could manifest as a small arrival time delay between the STARE2 detection at 1.4 GHz and the CHIME detection at lower frequencies.

The relative delay between the observed radio emission (corrected for DM) and its hard radiation counterpart observed at peak (Figure 8) should be, at most, comparable to the radio burst duration ( $\lesssim \mathrm{ms}$ in the case of SGR 1935+2154's April 28 burst). This results from the fact that the high-frequency photons are optically thin at the shock deceleration radius (when the emission peaks), while the radio is optically thick to induced scattering at this time and only escapes at a time $t \sim$ burst width later. Updated timing by Mereghetti et al. (2020a) suggests that the radio photons may in fact precede the X-ray peaks by a few milliseconds, which would be at odds with the above prediction. This is an important observation; however, we note that $\sim$ millisecond-resolution timing is currently very challenging based on limited X-ray photon counts and possible systematics associated with precision-level dedispersion of the radio bursts. Such dedispersion would require $\sim 0.03 \mathrm{pc} \mathrm{cm}^{-3}$ fidelity (with respect to the $\approx 332 \mathrm{pc} \mathrm{cm}^{-3}$ measured $\mathrm{DM}$ ) to achieve millisecond-level timing; however, the difference between the DMs quoted by CHIME and STARE2 is already comparable to this (and much larger than the quoted statistical uncertainties in either value). Furthermore, the value of the DM is known to depend on the dedispersion method used and can change by as much as $\sim 2 \mathrm{pc} \mathrm{cm}^{-3}$ between signal-to-noisemaximizing and "structure-maximizing" dedispersion methods (Hessels et al. 2019). We thus urge caution when interpreting such timing.

As shown in Figure 9, the shock model predicts that the X-ray emission should be accompanied by longer-lived synchrotron emission at lower frequencies as the shock propagates to larger radii, again similar to a GRB afterglow (Sari et al. 1998). For frequencies below $\nu_{\mathrm{syn}}$ and $\nu_{\mathrm{c}}$, the peak flux will rise as $F_{\nu} \propto \nu^{1 / 2}$ as $\nu_{\text {syn }} \propto t^{-\alpha}$ decreases in time until reaching the observing frequency, where $\alpha=3 / 2$ during the relativistic phase and $\alpha=3$ once the blast wave transitions to become nonrelativistic. Thus, if $\nu_{\text {syn }}$ is passing through the X-ray band $\left(\nu_{\mathrm{X}} \sim 10 \mathrm{keV}\right)$ on a timescale of $t_{\mathrm{X}} \sim 0.1 \mathrm{~s}$, it will reach the optical band on a timescale of seconds and the radio band $(\sim 10 \mathrm{GHz})$ on a timescale of less than $1 \mathrm{hr}$. For an external medium with a radially constant density, the flux density $F_{\nu}$ at peak $\left(\nu_{\mathrm{obs}} \sim \nu_{\mathrm{syn}}\right)$ is constant during the relativistic phase and thus approximately equal to that initially achieved at the higher frequencies. Using the X-ray fluence of $F_{X} \sim 7 \times 10^{-7}$ erg $\mathrm{cm}^{-2}$ of the FRB-generating flare from SGR $1935+2154$ as a

\footnotetext{
19 This phenomena, which is now well cataloged for many repeating FRB sources, has been termed the "sad trombone" (Hessels et al. 2019; CHIME/ FRB Collaboration et al. 2019).
} 


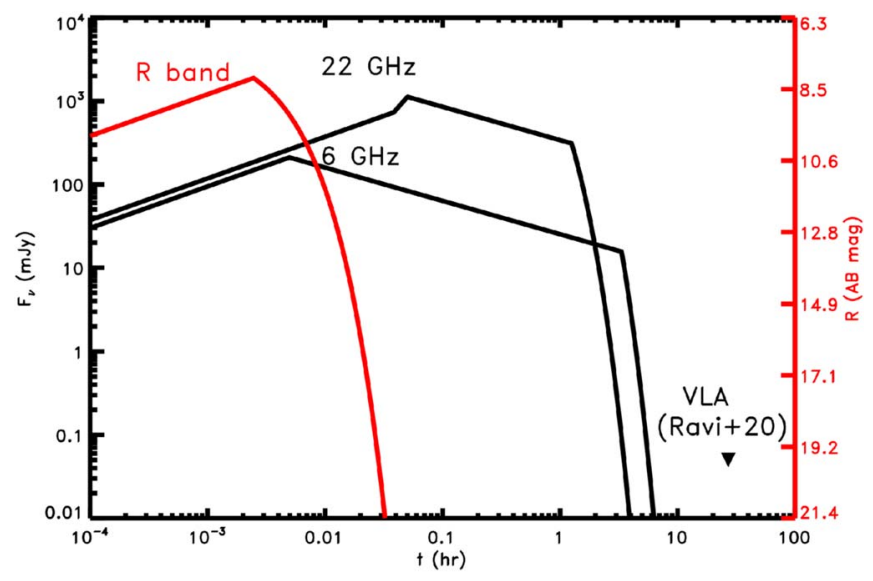

Figure 9. Optical $R$-band and $6 / 22 \mathrm{GHz}$ radio flux due to thermal synchrotron afterglow emission of the X-ray-/FRB-generated shock as a function of time in hours (bottom axis) as it propagates to larger radii. Shown for comparison are upper limits from Ravi et al. (2020a). In this simple estimate, we have assumed a radially constant density of the external medium; if the external medium does not extend so far, then the predicted flux would be lower than these estimates. Synchrotron self-absorption, which affects the radio light curves, has been included in an approximate way.

proxy for the latter, we predict a peak radio afterglow flux density $F_{\text {radio }} \sim F_{X} /\left(\nu_{\mathrm{X}} \cdot t_{\mathrm{X}}\right) \sim 0.3 \mathrm{Jy}$ (although self-absorption severely mitigates this estimate; see Figure 9). After peak, the predicted flux will decay exponentially as the synchrotron emission occurs from electrons on the Wien tail of the thermal Maxwellian. Thus, such an afterglow is consistent with upper limits on radio afterglow emission of $0.05 \mathrm{mJy}$ taken on a timescale of a few days (Ravi et al. 2020a, 2020b). Also note that the radius sampled by the shock at these late times is a factor of $\gtrsim 30$ times larger than at the time of the earlier radio emission, and there is no guarantee that the dense medium extends that far from the magnetar.

Finally, the ejection of the baryonic outflow is predicted to result in a small decrease in the spin frequency of the magnetar due to the temporary opening up of field lines by the massloaded wind (Thompson \& Blaes 1998; Harding et al. 1999; Beniamini et al. 2020). The magnitude of the spin frequency change increases with the luminosity of the baryonic wind and its duration, both of which are highly uncertain. However if the $\mathrm{X}$-ray burst concurrent with the FRB produces such an outflow, then there are at least two other bursts within the latest outburst, with even larger luminosities (Ridnaia et al. 2020b; Veres et al. 2020), that may have also produced an outflow. Taking, for example, the brightest of these bursts, which occurred on April 22 , we can calculate a lower limit on the spin frequency decrease of $\Delta \Omega=2 \times 10^{-8} L_{41}^{1 / 2} t^{1 / 2} \mathrm{~s}^{-1}$ (Beniamini et al. 2020).

The amount of baryonic matter necessary to explain the observed radio burst within this version of the synchrotron maser model is very small, $M_{\mathrm{b}} \sim 4 \pi r_{\mathrm{sh}}^{3} n_{\mathrm{ext}} m_{p} / 3 \sim 4 \times 10^{14} \mathrm{~g}$, although the baryon shell may extend to radii $r_{\mathrm{b}} \gg r_{\mathrm{sh}}$, in which case the mass estimate would increase by $\sim\left(r_{\mathrm{b}} / r_{\mathrm{sh}}\right)^{3}$. The energy associated with this shell is only $\sim M_{\mathrm{b}} v_{\mathrm{b}}^{2} / 2 \sim$ $4 \times 10^{34} \mathrm{erg}$, where we have assumed a characteristic expansion velocity for the baryonic shell of $v_{\mathrm{b}}=0.5 c$, which also implies that the shell must have been ejected $\gtrsim r_{\mathrm{sh}} / v_{\mathrm{b}} \sim 10 \mathrm{~s}$ prior to the radio burst. Although the luminosity of the X-ray bursts from SGR $1935+2154$ does not typically surpass the magnetic Eddington limit (Figure 1), the physics of baryon ejection from active magnetars is still uncertain, and there may be alternative ejection mechanisms that can provide the necessary environment. The modest amount of material required in this baryon shell implies that, despite the uncertainty in the details, the ejection process is energetically unprohibitive for active magnetars.

\subsubsection{Spin-down-powered Wind (Beloborodov 2017, 2020)}

Beloborodov $(2017,2020)$ argued that the upstream medium into which the relativistic flare collides is an electron/positron plasma from a spin-down-powered component to the pulsar wind. Given the low spin-down power of SGR 1935 +2154 , however, this scenario might be somewhat disfavored.

The observed radio fluence at $\nu=1.4 \mathrm{GHz}$ in this model can be estimated from Equation (91) of Beloborodov (2020) and reexpressed as a function of the spin-down power $L_{\mathrm{sd}}$ and pair multiplicity $\mathcal{M}$,

$$
E_{\text {radio }} \sim 3 \times 10^{31} \operatorname{erg} L_{\text {sd, } 34}^{1 / 12} \mathcal{M}_{3}^{2 / 3} .
$$

In the above, we have assumed that the flare energy is $10^{40} \mathrm{erg}$, motivated by the X-ray counterpart of SGR $1935+2154$, and we have omitted scaling with nuisance parameters for clarity. For the spin-down power of SGR $1935+2154$ and standard pair multiplicity of $10^{3}$, this fluence is $\sim 3$ orders of magnitude lower than the observed energy of SGR 1935+2154's radio burst.

This tension may be alleviated by an enhancement of the magnetar wind (because of, e.g., opening of field lines by the magnetar flare) or an increased pair multiplicity shortly preceding the flare (Beloborodov 2020). From Equation (18), we find that a pair multiplicity of $\sim 5 \times 10^{7}$ would be required to fit the observed fluence. This is much higher than pulsar pair multiplicities, though it has been suggested that much larger values $\gg 10^{3}$ may be attainable for magnetars immediately preceding a flare (Beloborodov 2020).

The high pair loading in this version of the synchrotron maser model, as in the Lyubarsky (2014) model, pushes the high-energy thermal synchrotron counterpart to lower frequencies $\left(\nu_{\text {syn }} \propto f_{\mathrm{e}}^{-2}\right)$, leading to an afterglow peaking optical/UV band (Beloborodov 2020) instead of the X-ray/gamma-ray band predicted in the baryonic model (Figure 8). In this scenario, as in the magnetar wind nebula case (Section 5.2.1), the X-rays from the flare must be created by a process that is not directly related to the FRB mechanism.

\subsection{Additional Models}

In curvature models, the FRB is produced by curvature radiation from bunched electrons streaming along the magnetic field lines of the magnetar (e.g., Kumar et al. 2017; Lu \& Kumar 2018). These models predict fluence ratios $\eta \sim 1$ in all bands (Chen et al. 2020) and thus cannot explain the properties of the observed X-ray burst of SGR 1935+2154 (whose fluence ratio is $\eta \sim 10^{-5} \ll 1$, but also in terms of $\mathrm{X}$-ray spectrum) as a bona fide FRB counterpart. This shortcoming can potentially be dismissed by arguing that the same magnetar activity leading to particle bunching, acceleration, and FRB emission also produces "normal" short X-ray bursts. However, this scenario makes no prediction as to the quantitative relationship between the radio and X-ray burst properties, and the observed fluence ratio of $\eta \sim 10^{-5}$ is ad hoc within this framework. These considerations also apply to models where 
FRBs are produced by reconnection in the outer magnetosphere (Lyubarsky 2020) or, indeed, to any magnetospheric models. If true, a testable prediction of the scenario (and one that would be in tension with synchrotron maser models) may be large variations in the value of $\eta$ for future events.

Another class of FRB models discussed in the literature power FRBs by the kinetic energy of an outflow interacting with the magnetosphere of an NS (Zhang 2017, 2018; Ioka \& Zhang 2020). The outflow in these "cosmic comb" models has been suggested to range from AGN-driven winds to GRB jets or winds from a binary companion to the NS. None of the above are likely to be applicable in the context of SGR 1935 +2154 's radio burst, in tension with predictions of this model (although see Wang et al. 2020).

A final class of NS-related models we discuss are the "spindown models," in which the radio bursts are powered by rotational energy of the NS (Connor et al. 2016; Cordes \& Wasserman 2016; Muñoz et al. 2020). Though in principle applicable to magnetars, these models typically envision normal pulsars as progenitors. Indeed, considering the very low spin-down power of SGR $1935+2154$ in comparison to pulsars, and the fact that the latter are $\gtrsim 100$ times more common than Galactic magnetars (in terms of their effective active lifetime), it seems unnatural that a spin-down-powered FRB would be first detected for SGR $1935+2154$. A prediction of this model would be a change in NS spin period due to the released burst energy. Accounting for the radiated energy of SGR 1935+2154's burst, including its associated X-ray counterpart, we find that a change $\Delta \Omega \approx-4 \times 10^{-6} \mathrm{~s}^{-1} d_{10}^{2}$ in the angular velocity of SGR $1935+2154$ needs to have occurred. A reduction in the spin frequency is also expected in the Metzger et al. (2019) version of the synchrotron maser model due to the opening of magnetic field lines by the baryonic outflow; however, this effect is much smaller (Section 5.2.2). The Beloborodov (2020) synchrotron maser model (Section 5.2.3) also necessitates some level of period change due to a significant enhancement of the pulsar wind required immediately preceding the FRB; however, this too would be expected to be smaller than the $\Delta \Omega$ estimated above. Future timing of SGR 1935+2154 may help test this prediction of the spin-down model.

\section{Conclusions}

We have explored implications related to the population of FRB sources and to theoretical models of FRBs in light of the recent detection of a luminous millisecond radio burst from the Galactic magnetar SGR 1935+2154 (Bochenek et al. 2020c; CHIME/FRB Collaboration et al. 2020b) in conjunction with an X-ray counterpart (Li et al. 2020; Mereghetti et al. 2020a; Ridnaia et al. 2020e; Tavani et al. 2020). The large energy of this burst makes it unique among any previously observed pulsar/magnetar phenomenology and bridges the gap to extragalactic FRBs (Bochenek et al. 2020c; CHIME/FRB Collaboration et al. 2020b).

With regard to the general implications for extragalactic magnetar populations, our conclusions may be summarized as follows.

1. Broadly speaking, the discovery of a highly luminous millisecond-duration radio burst coincident with the X-ray flare of the Galactic magnetar SGR $1935+2154$ supports magnetar models for extragalactic FRBs.
2. The X-ray properties of the flare are fairly typical of Galactic magnetar flares in terms of fluence and overall duration (Figure 1); however, its X-ray spectrum may have been harder than usual (Mereghetti et al. 2020a; Ridnaia et al. 2020e). Furthermore, with the notable exception of the giant flare from SGR 1806-20, relatively few such flares would have previously been detected at radio wavelengths for a similar low ratio $\eta \equiv E_{\text {radio }} / E_{\mathrm{X}} \sim 10^{-5}$ of radio to $\mathrm{X}$-ray fluence (Figure 2). This suggests that a sizable fraction of Galactic magnetar flares may be accompanied by luminous radio bursts, although we caution that multiple emission mechanisms may be at play in producing magnetar X-ray flares, and that only a subset (e.g., those produced by shocks; see the subsequent set of bullets and Section 5.2.2) may be expected to emit coherent radio bursts.

3. Applying the same fluence ratio $\eta$ to giant magnetar flares would imply that Galactic magnetars are capable of powering even the most energetic cosmological FRBs (Figure 3). However, a stark discrepancy exists between the activity (burst repetition rate) of Galactic magnetars and the sources of the recurring extragalactic FRBs. If universal, the low efficiency $\eta \sim 10^{-5}$ also places strong upper limits on the magnetar active lifetime in the latter case, much shorter than the ages of most Galactic magnetars (Equation (2)).

4. The estimated rate of radio bursts similar to that observed from SGR $1935+2154$ is insufficient to contribute appreciably to the observed extragalactic FRB rate (Equation (3)). Depending on the luminosity function of the Galactic flares, the all-sky FRB rate (also including giant flares) can be reproduced by ordinary Galactic magnetars similar to SGR $1935+2154$ (Figure 4). However, such a model fails to simultaneously explain the large (per-source) repetition rate of known repeaters or the large DMs of the FRB population.

5. Instead, considering a two-component model, we add a second population of magnetars whose birth rate is a free parameter and whose activity level and lifetime are scaled from SGR $1935+2154$ as a function of the population's magnetic field strength. This model allows one to broadly replicate the observed properties of the FRB population (Figure 6), but only if the birth rate of the active magnetar population is «the CCSN rate (Figure 5; in line with previous work, e.g., Nicholl et al. 2017).

6. This implies that the population of active magnetars cannot be interpreted as an earlier evolutionary state of SGR 1935+2154-like magnetars, which are born in a large fraction of CCSNe. Instead, this population may form through more exotic channels, such as SLSNe, AIC, or binary NS mergers (Metzger et al. 2017; Margalit et al. 2019).

In addition to the general implications summarized above, we also address implications for specific FRB magnetar models. We stress that there is no single magnetar model for FRBs, many distinct models have been suggested in the literature, and these differ in the requisite magnetar properties, FRB emission mechanism, and predictions for (or lack of) multiwavelength counterparts (Section 5). In this context, we find the following.

1. Magnetospheric models (curvature, low-twist, and reconnection models) predict either no high-energy counterpart 
or weak counterparts of comparable energy to the radio emission $(\eta \sim 1)$. This is in tension with the observed X-ray counterpart in SGR 1935+2154, where $\eta \sim 10^{-5}$. This shortcoming can be dismissed by interpreting the X-ray counterpart as a normal short X-ray burst. However, this scenario makes no predictions of the spectral or energetic properties of the X-ray flare, and the $\eta \sim 10^{-5}$ radio-to-X-ray fluence ratio is ad hoc within this framework (Section 5.3).

2. Synchrotron maser models, which involve relativistic flare ejecta colliding with an external medium, provide a promising alternative. However, these models differ in the nature of the upstream medium and their predicted multiwavelength afterglow.

3. Models in which the upstream is the magnetar wind nebula (Lyubarsky 2014; Section 5.2.1) may have an efficiency issue and predict associated afterglow in the $\mathrm{GeV}$ range. Likewise, models in which the upstream is a rotational-powered pulsar wind (Beloborodov 2017, 2020) are strained and predict a lower-frequency counterpart (Section 5.2.3).

4. The baryonic shell version of the synchrotron maser model naturally explains the value of $\eta$ (compare Equations (1) and 14) in addition to the timing ${ }^{20}$ and spectral features of the observed X-ray emission (Figure 8). The model requires substantial mass ejection to accompany the flares, which may be in tension with the relatively low luminosity of the flares if the outflows are driven by radiation pressure (Figure 2). On the other hand, the requirement for mass ejection-and the sensitivity of the radio emission to the detailed properties of the upstream medium-could help explain why not all magnetar flares are accompanied by a luminous FRB.

5. The latter model suggests a new paradigm in which X-ray emission of magnetar flares arises from thermal synchrotron radiation from internal shocks. This model predicts a time-resolved spectrum that follows an exponentially cutoff power law and correlations between the peak energy of the burst and other burst properties (e.g., duration and fluence), which are broadly consistent with observations (van der Horst et al. 2012).

6. The baryon shell synchrotron maser shock model (Metzger et al. 2019; Margalit et al. 2020) makes several predictions testable by future Galactic or extragalactic FRBs. (1) Although X-ray/gamma-ray emission can arise from magnetar flares without an accompanying FRB (e.g., if the X-rays/gamma-rays do not arise from shocks), the opposite is not true. Any FRB-like burst should be accompanied by X-ray/gamma-ray emission with energy at least a factor of $\eta^{-1} \gtrsim 10^{4}$ larger than the emitted radio energy (Equation (14)). (2) Scaling up to cosmological FRBs, the equivalent prompt synchrotron counterpart should peak in the $\gtrsim \mathrm{MeV}-\mathrm{GeV}$ gamma-ray band (Figure 8). (3) Galactic FRBs may be accompanied by longer-lived optical/radio emission on a timescale of seconds/minutes (Figure 9), the details of which, however, depend on the extent of the external medium surrounding the magnetar on larger radial scales than probed by the hard X-rays. (4) The radio and hard X-ray

\footnotetext{
${ }^{20}$ Updated millisecond-level timing suggests a possible tension (Mereghetti et al. 2020a); however, we note that such timing may be affected by nontrivial systematics (see Section 5.2.2).
}

bursts are predicted to be jointly beamed within this model (which is not necessarily the case in other models). The relativistic plasmoid that produces the shock must therefore point toward the observer. If this directionality is linked to the magnetic structure of the NS, it is possible that FRB-producing bursts occur at a common rotational phase (although the pulse fraction may be quite large if the beaming fraction of $\sim \Omega_{\mathrm{pl}} / 4 \pi$ is not too small, where $\Omega_{\mathrm{pl}}$ is the solid angle subtended by the plasmoid; see, e.g., Yuan et al. 2020).

We thank Bryan Gaensler and George Younes for helpful insight. B.M. is supported by NASA through NASA Hubble Fellowship grant No. HST-HF2-51412.001-A awarded by the Space Telescope Science Institute, which is operated by the Association of Universities for Research in Astronomy, Inc., for NASA under contract NAS5-26555. The research of P.B. was funded by the Gordon and Betty Moore Foundation through grant GBMF5076. N.S. is supported by the Columbia University Dean's fellowship and through the National Science Foundation (grant No. 80NSSC18K1104). B.D.M. is supported in part by the Simons Foundation through the Simons Fellowship Program in Mathematics and Physics (grant No. 606260).

\section{ORCID iDs}

Ben Margalit (iD https://orcid.org/0000-0001-8405-2649

Paz Beniamini (1) https://orcid.org/0000-0001-7833-1043

Navin Sridhar (1D https://orcid.org/0000-0002-5519-9550

Brian D. Metzger (iD https://orcid.org/0000-0002-4670-7509

\section{References}

Bandura, K., Addison, G. E., Amiri, M., et al. 2014, Proc. SPIE, 9145, 914522 Bannister, K. W., Deller, A. T., Phillips, C., et al. 2019, Sci, 365, 565

Baring, M. G., Wadiasingh, Z., \& Gonthier, P. L. 2011, ApJ, 733, 61

Bassa, C. G., Tendulkar, S. P., Adams, E. A. K., et al. 2017, ApJL, 843, L8 Beloborodov, A. M. 2017, ApJL, 843, L26

Beloborodov, A. M. 2020, ApJ, 896, 142

Beloborodov, A. M., \& Li, X. 2016, ApJ, 833, 261

Beniamini, P., \& Giannios, D. 2017, MNRAS, 468, 3202

Beniamini, P., Hotokezaka, K., van der Horst, A., \& Kouveliotou, C. 2019, MNRAS, 487, 1426

Beniamini, P., Wadiasingh, Z., \& Metzger, B. D. 2020, arXiv:2003.12509 Bhandari, S., Keane, E. F., Barr, E. D., et al. 2018, MNRAS, 475, 1427

Bhandari, S., Sadler, E. M., Prochaska, J. X., et al. 2020, ApJL, 895, L37

Blanchard, P. K., Berger, E., \& Fong, W.-f. 2016, ApJ, 817, 144

Bochenek, C., Kulkarni, S., Ravi, V., et al. 2020a, ATel, 13684, 1

Bochenek, C. D., McKenna, D. L., Belov, K. V., et al. 2020b, PASP, 132, 034202

Bochenek, C. D., Ravi, V., Belov, K. V., et al. 2020c, arXiv:2005.10828

Borghese, A., Coti Zelati, F., Rea, N., et al. 2020, arXiv:2006.00215

Burgay, M., Israel, G. L., Rea, N., et al. 2014, ATel, 6371, 1

Chatterjee, S., Law, C. J., Wharton, R. S., et al. 2017, Natur, 541, 58

Chen, G., Ravi, V., \& Lu, W. 2020, ApJ, 897, 146

Cheng, Y., Zhang, G. Q., \& Wang, F. Y. 2020, MNRAS, 491, 1498

Cherry, M. L., Yoshida, A., Sakamoto, T., et al. 2020, GCN, 27623, 1

CHIME/FRB Collaboration, Andersen, B. C., Bandura, K., et al. 2019, ApJL, $885, \mathrm{~L} 24$

Colpi, M., Geppert, U., \& Page, D. 2000, ApJL, 529, L29

Connor, L., Sievers, J., \& Pen, U.-L. 2016, MNRAS, 458, L19

Cordes, J. M., \& Chatterjee, S. 2019, ARA\&A, 57, 417

Cordes, J. M., \& Wasserman, I. 2016, MNRAS, 457, 232

Dall'Osso, S., Granot, J., \& Piran, T. 2012, MNRAS, 422, 2878

de Luca, A., Caraveo, P. A., Mereghetti, S., Tiengo, A., \& Bignami, G. F. 2006, Sci, 313, 814

Eftekhari, T., Berger, E., Williams, P. K. G., \& Blanchard, P. K. 2018, ApJ, 860, 73

Feroci, M., Mereghetti, S., Woods, P., et al. 2003, ApJ, 596, 470 
Fruchter, A. S., Levan, A. J., Strolger, L., et al. 2006, Natur, 441, 463 Gaensler, B. M. 2014, GCN, 16533, 1

Gallant, Y. A., Hoshino, M., Langdon, A. B., Arons, J., \& Max, C. E. 1992, ApJ, 391, 73

Gavriil, F. P., Kaspi, V. M., \& Woods, P. M. 2004, ApJ, 607, 959

Gehrels, N. 1986, ApJ, 303, 336

Gelfand, J. D., Lyubarsky, Y. E., Eichler, D., et al. 2005, ApJL, 634, L89

Giannios, D., \& Spitkovsky, A. 2009, MNRAS, 400, 330

Göğüş, E., Kouveliotou, C., Woods, P. M., et al. 2001, ApJ, 558, 228

Göğüş, E., Woods, P. M., Kouveliotou, C., et al. 1999, ApJL, 526, L93

Göğüş, E., Woods, P. M., Kouveliotou, C., et al. 2000, ApJL, 532, L121

Göğüş, E., Woods, P. M., Kouveliotou, C., et al. 2011, ApJ, 740, 55

Gourdji, K., Michilli, D., Spitler, L. G., et al. 2019, ApJL, 877, L19

Granot, J., Gill, R., Younes, G., et al. 2017, MNRAS, 464, 4895

Granot, J., Ramirez-Ruiz, E., Taylor, G. B., et al. 2006, ApJ, 638, 391

Harding, A. K., Contopoulos, I., \& Kazanas, D. 1999, ApJL, 525, L125

Hessels, J. W. T., Spitler, L. G., Seymour, A. D., et al. 2019, ApJL, 876, L23

Hopkins, A. M., \& Beacom, J. F. 2006, ApJ, 651, 142

Hurley, K., Boggs, S. E., Smith, D. M., et al. 2005, Natur, 434, 1098

Hurley, K., Mitrofanov, I. G., Golovin, D., et al. 2020, GCN, 27625, 1

Ioka, K., \& Zhang, B. 2020, ApJL, 893, L26

Israel, G. L., Esposito, P., Rea, N., et al. 2016, MNRAS, 457, 3448

Israel, G. L., Rea, N., Zelati, F. C., et al. 2014, ATel, 6370, 1

Iwamoto, M., Amano, T., Hoshino, M., et al. 2019, ApJL, 883, L35

Katz, J. I. 2016, ApJ, 826, 226

Katz, J. I. 2020, MNRAS, 494, L64

Kennea, J. A., Beardmore, A. P., Page, K. L., \& Palmer, D. M. 2020, ATel, 13679,1

Kothes, R., Sun, X., Gaensler, B., \& Reich, W. 2018, ApJ, 852, 54

Kozlova, A. V., Israel, G. L., Svinkin, D. S., et al. 2016, MNRAS, 460, 2008

Kulkarni, S. R., Ofek, E. O., Neill, J. D., Zheng, Z., \& Juric, M. 2014, ApJ, 797, 70

Kumar, P., Lu, W., \& Bhattacharya, M. 2017, MNRAS, 468, 2726

Law, C. J., Abruzzo, M. W., Bassa, C. G., et al. 2017, ApJ, 850, 76

Levin, Y., Beloborodov, A. M., \& Bransgrove, A. 2020, ApJL, 895, L30

Li, C. K., Lin, L., Xiong, S. L., et al. 2020, arXiv:2005.11071

Li, W., Chornock, R., Leaman, J., et al. 2011, MNRAS, 412, 1473

Li, Y., \& Zhang, B. 2020, ApJL, 899, L6

Lien, A. Y., Barthelmy, S. D., Baumgartner, W. H., et al. 2014, GCN, 16522, 1

Lin, L., Gogus, E., Roberts, O. J., et al. 2020a, ApJ, 893, 156

Lin, L., Zhang, C. F., Wang, P., et al. 2020b, arXiv:2005.11479

Linscott, I. R., \& Erkes, J. W. 1980, ApJL, 236, L109

Lipunov, V., Zhirkov, K., Kornilov, V., et al. 2020, GCN, 27670, 1

Lithwick, Y., \& Sari, R. 2001, ApJ, 555, 540

Lorimer, D. R., Bailes, M., McLaughlin, M. A., Narkevic, D. J., \& Crawford, F. 2007, Sci, 318, 777

Lu, W., \& Kumar, P. 2018, MNRAS, 477, 2470

Lu, W., \& Phinney, E. S. 2020, MNRAS, 496, 3308

Lu, W., \& Piro, A. L. 2019, ApJ, 883, 40

Lunnan, R., Chornock, R., Berger, E., et al. 2015, ApJ, 804, 90

Lyubarsky, Y. 2014, MNRAS, 442, L9

Lyubarsky, Y. 2020, ApJ, 897, 1

Lyutikov, M., Barkov, M. V., \& Giannios, D. 2020, ApJL, 893, L39

Marathe, A., Shenoy, V., Bhalerao, V., et al. 2020, GCN, 27664, 1

Marcote, B., Nimmo, K., Hessels, J. W. T., et al. 2020, Natur, 577, 190

Margalit, B., Berger, E., \& Metzger, B. D. 2019, ApJ, 886, 110

Margalit, B., Metzger, B. D., \& Sironi, L. 2020, MNRAS, 494, 4627

Masui, K., Lin, H.-H., Sievers, J., et al. 2015, Natur, 528, 523

Mazets, E. P., Cline, T. L., Aptekar, R. L., et al. 1999, AstL, 25, 635

Mereghetti, S., Götz, D., Weidenspointner, G., et al. 2009, ApJL, 696, L74

Mereghetti, S., Savchenko, V., Ferrigno, C., et al. 2020a, ApJL, 898, L29

Mereghetti, S., Savchenko, V., Gotz, D., et al. 2020b, GCN, 27668, 1

Metzger, B. D., Berger, E., \& Margalit, B. 2017, ApJ, 841, 14

Metzger, B. D., Margalit, B., \& Sironi, L. 2019, MNRAS, 485, 4091

Michilli, D., Seymour, A., Hessels, J. W. T., et al. 2018, Natur, 553, 182

Mickaliger, M. B., McLaughlin, M. A., Lorimer, D. R., et al. 2012, ApJ, 760,64

Muñoz, J. B., Ravi, V., \& Loeb, A. 2020, ApJ, 890, 162

Nicholl, M., Williams, P. K. G., Berger, E., et al. 2017, ApJ, 843, 84
Olausen, S. A., \& Kaspi, V. M. 2014, ApJS, 212, 6

Paczynski, B. 1992, AcA, 42, 145

Palmer, D. M. 2020, ATel, 13675, 1

Palmer, D. M., Barthelmy, S., Gehrels, N., et al. 2005, Natur, 434, 1107

Pavlović, M. Z., Urošević, D., Vukotić, B., Arbutina, B., \& Göker, Ü D. 2013, ApJS, 204, 4

Perley, D. A., Krühler, T., Schady, P., et al. 2017, MNRAS, 465, L89

Petroff, E., Hessels, J. W. T., \& Lorimer, D. R. 2019, A\&ARv, 27, 4

Platts, E., Weltman, A., Walters, A., et al. 2019, PhR, 821, 1

Plotnikov, I., \& Sironi, L. 2019, MNRAS, 485, 3816

Popov, S. B., \& Postnov, K. A. 2013, arXiv:1307.4924

Prochaska, J. X., Macquart, J.-P., McQuinn, M., et al. 2019, Sci, 366, 231

Ravi, V. 2019, NatAs, 3, 928

Ravi, V., Catha, M., D’Addario, L., et al. 2019, Natur, 572, 352

Ravi, V., Hallinan, G., \& Law, C. J. 2020a, ATel, 13690, 1

Ravi, V., Hallinan, G., \& Law, C. J. 2020b, ATel, 13693, 1

Ricciarini, S., Yoshida, A., Sakamoto, T., et al. 2020, GCN, 27663, 1

Ridnaia, A., Golenetskii, S., Aptekar, R., et al. 2020a, GCN, 27554, 1

Ridnaia, A., Golenetskii, S., Aptekar, R., et al. 2020b, GCN, 27631, 1

Ridnaia, A., Golenetskii, S., Aptekar, R., et al. 2020c, GCN, 27667, 1

Ridnaia, A., Golenetskii, S., Aptekar, R., et al. 2020d, GCN, 27669, 1

Ridnaia, A., Svinkin, D., Frederiks, D., et al. 2020e, arXiv:2005.11178

Sari, R., Piran, T., \& Narayan, R. 1998, ApJL, 497, L17

Scholz, P. \& CHIME/FRB Collaboration 2020, ATel, 13681, 1

Shannon, R. M., Macquart, J. P., Bannister, K. W., et al. 2018, Natur, 562, 386

Simard, D., \& Ravi, V. 2020, arXiv:2006.13184

Sironi, L., \& Spitkovsky, A. 2011, ApJ, 726, 75

Spitler, L. G., Scholz, P., Hessels, J. W. T., et al. 2016, Natur, 531, 202

Stamatikos, M., Malesani, D., Page, K. L., \& Sakamoto, T. 2014, GCN, 16520,1

Sun, X. H., Reich, P., Reich, W., et al. 2011, A\&A, 536, A83

Surnis, M. P., Joshi, B. C., Maan, Y., et al. 2016, ApJ, 826, 184

Surnis, M. P., Krishnakumar, M. A., Maan, Y., Joshi, B. C., \& Manoharan, P. K. 2014, ATel, 6376, 1

Svinkin, D., Golenetskii, S., Aptekar, R., et al. 2020, GCN, 27527, 1

Tanaka, Y. T., Terasawa, T., Kawai, N., et al. 2007, ApJL, 665, L55

Tavani, M., Casentini, C., Ursi, A., et al. 2020, arXiv:2005.12164

Tendulkar, S. P., Bassa, C. G., Cordes, J. M., et al. 2017, ApJL, 834, L7

Tendulkar, S. P., Cameron, P. B., \& Kulkarni, S. R. 2012, ApJ, 761, 76

Tendulkar, S. P., Kaspi, V. M., \& Patel, C. 2016, ApJ, 827, 59

The CHIME/FRB Collaboration, Amiri, M., Andersen, B. C., et al. 2020a, Natur, 7812, 351

The CHIME/FRB Collaboration, Andersen, B. C., Bandura, K. M., et al. 2020b, arXiv:2005.10324

Thompson, C., \& Blaes, O. 1998, PhRvD, 57, 3219

Thornton, D., Stappers, B., Bailes, M., et al. 2013, Sci, 341, 53

Tong, H., Wang, W., \& Wang, H. G. 2020, arXiv:2002.10265

van der Horst, A. J., Kouveliotou, C., Gorgone, N. M., et al. 2012, ApJ, 749,122

Vandenbroucke, J. 2020, ATel, 13689, 1

Veres, P., Bissaldi, E., Briggs, M. S. \& Fermi GBM Team 2020, GCN, 27531, 1

Wadiasingh, Z., Beniamini, P., Timokhin, A., et al. 2020, ApJ, 891, 82

Wadiasingh, Z., \& Timokhin, A. 2019, ApJ, 879, 4

Wang, W.-Y., Xu, R., Zheng, X., \& Chen, X. 2020, arXiv:2005.02100

Yang, H., \& Zou, Y.-C. 2020, ApJL, 893, L31

Younes, G., Gogus, E., Kouveliotou, C., \& van der Hors, A. J. 2015, ATel, 7213, 1

Younes, G., Guver, T., Enoto, T., et al. 2020, ATel, 13678, 1

Younes, G., Kouveliotou, C., Jaodand, A., et al. 2017, ApJ, 847, 85

Yuan, Y., Beloborodov, A. M., Chen, A. Y., \& Levin, Y. 2020, arXiv:2006. 04649

Zanazzi, J. J., \& Lai, D. 2020, ApJL, 892, L15

Zhang, B. 2017, ApJL, 836, L32

Zhang, B. 2018, ApJL, 854, L21

Zhang, S. N., Tuo, Y. L., Xiong, S. L., et al. 2020a, ATel, 13687, 1

Zhang, S. N., Xiong, S. L., Li, C. K., et al. 2020b, ATel, 13696, 1

Zhong, S.-Q., \& Dai, Z.-G. 2020, ApJ, 893, 9

Zhou, P., Zhou, X., Chen, Y., et al. 2020, arXiv:2005.03517 